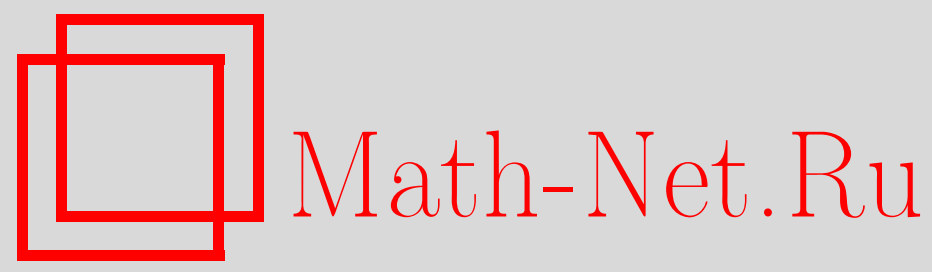

И. П. Чухров, О тупиковых комплексах граней в единичном кубе, Дискрет. матем., 2011, том 23, выпуск 1, 132-158

DOI: https://doi.org/10.4213/dm1136

Использование Общероссийского математического портала Math-Net.Ru подразумевает, что вы прочитали и согласны с пользовательским соглашением http://www.mathnet.ru/rus/agreement

Параметры загрузки:

IP: 54.197 .217 .227

26 апреля 2023 г., 18:21:03 


\title{
О тупиковых комплексах граней в единичном кубе
}

\author{
() 2011 г. . П. Чухров
}

\begin{abstract}
Изучение свойств тупиковых комплексов граней связано с задачей минимизации булевых функций в классе дизъюнктивных нормальных форм (д.н.ф.). В работах С. В. Яблонского, Ю. И. Журавлева, В. В. Глаголева, Ю. Л. Васильева, А. А. Сапоженко на основе построения и исследования свойств конкретных булевых функций были получены оценки максимальных значений длины и числа тупиковых д.н.ф.

Автором был предложен другой подход, основанный на построении и оценивании мощности множеств тупиковых комплексов граней. В данной статье, с использованием вероятностного подхода, усовершенствованы методы построения и оценивания характеристик тупиковых комплексов граней, что позволило улучшить известные ранее оценки. На основе метода построения тупиковых комплексов граней в поясе единичного куба $B^{n}$ ширины $k$ получены оценки максимального числа граней и числа тупиковых комплексов для граней размерности $k<(1 / 4-\varepsilon) n$, где $\varepsilon-$ сколь угодно малая положительная постоянная. За счет оптимального выбора параметров для логарифма числа тупиковых комплексов граней получена нижняя оценка порядка $n 2^{n} \mathrm{c}$ константой $1,355 \cdot 2^{-5}$ при размерности граней $k \approx 0,0526 n$.

В силу эквивалентности задачи минимизации булевых функций в классе д.н.ф. и задачи построения комплексов граней, покрывающих подмножества вершин единичного куба, полученные результаты справедливы для оценки максимальных значений длины и числа тупиковых д.н.ф.
\end{abstract}

\section{1. Введение}

Исследование комплексов граней в единичном кубе, которые обладают экстремальными значениями таких характеристик, как число граней и число тупиковых комплексов, непосредственно связано с задачей минимизации булевых функций в классе дизъюнктивных нормальных форм (далее д.н.ф.) [1, 2, 3].

Геометрическая интерпретация задачи минимизации булевых функций основана на соответствии между вершинами $n$-мерного единичного куба $B^{n}$, входящими в множество единичных вершин функции, и слагаемыми совершенной д.н.ф. функции $f \in P_{n}$, с одной стороны, и между гранями единичного куба и импликантами, с другой стороны. Задаче построения тупиковой, кратчайшей или минимальной д.н.ф. соответствует задача построения покрытия подмножества вершин единичного куба комплексом граней, обладающего соответствующими свойствами.

Задача о поведении максимальных значений числа тупиковых и минимальных д.н.ф. булевой функции с ростом числа переменных была поставлена С. В. Яблонским в связи 
с оцениванием трудоемкости минимизации булевых функций алгоритмами, гарантирующими построение тупиковых д.н.ф. (см. с. 307 в [4]).

Характерной чертой задачи минимизации является возможность построения оптимального решения путем применения простых упрощений локального характера в сочетании с ярко выраженной полиэкстремальностью. Как правило, имеется большое число локальных экстремумов, роль которых играют тупиковые д.н.ф., среди которых отыскиваются глобальные экстремумы, то есть минимальные по некоторой мере сложности д.н.ф. Построение некоторого локального экстремума осуществляется относительно просто, при этом основная трудность заключается в выборе стратегии поиска глобальных экстремумов среди огромного числа локальных.

При изложении будем использовать понятия и обозначения, связанные с гранями и покрытиями подмножеств вершин единичного куба $B^{n}$, в следующей геометрической интерпретации.

Множество всех вершин куба $B^{n}$, которое совпадает с $k$-мерной гранью куба $B^{n}$, эквивалентно множеству всех вершин, на которых некоторая импликанта ранга $n-k$ обращается в единицу. Множество вершин грани также может быть представлено в виде $\left\{\tilde{x} \in B^{n}: \tilde{\alpha} \leqslant \tilde{x} \leqslant \tilde{\beta}\right\}$, где $\tilde{\alpha}$ и $\tilde{\beta}-$ минимальная и максимальная вершины грани, при этом расстояние Хэмминга $\rho(\tilde{\alpha}, \tilde{\beta})=k$. В таком представлении множество вершин грани называется $k$-мерным интервалом (интервалом размерности $k$ ) в единичном кубе $B^{n}$.

Комплекс граней

$$
T=\left\{I_{r}, r=1, \ldots, l\right\},
$$

покрывающий множество вершин

$$
N_{T}=\bigcup_{r=1}^{l} I_{r} \subseteq B^{n},
$$

называется тупиковым, если любая грань $I_{r} \in T$ является максимальной для множества $N_{T}$ и не покрывается остальными гранями из $T$. Число граней в произвольном комплексе $T$ будем обозначать $l(T)$. Вершины, которые в тупиковом комплексе $T$ покрываются только одной гранью, называются собственными. Каждая грань в тупиковом комплексе имеет хотя бы одну собственную вершину. Будем обозначать $C_{T}$ подмножество собственных вершин тупикового комплекса $T$, которое содержит по одной произвольной, если их несколько, собственной вершине для каждой грани из тупикового комплекса. Для тупикового комплекса граней

$$
l(T)=\left|C_{T}\right|
$$

Комплексу граней $T$, покрывающему множество вершин $N_{T}$, однозначно соответствует функция $f \in P_{n}$, для которой $N_{f}=N_{T}$ и д.н.ф., которая состоит из импликант, однозначно определяемых гранями комплекса $T$. Тупиковому комплексу граней соответствует тупиковая д.н.ф. функции.

Подмножество вершин единичного куба, состоящее из слоев $B_{i}^{n}$ с номерами $i=m-k, \ldots, m$, называется поясом в кубе $B^{n}$ и обозначается

$$
\begin{aligned}
S_{m-k, m}^{n} & =\bigcup_{i=m-k}^{m} B_{i}^{n} \\
& =\left\{\tilde{x} \in B^{n}: m-k \leqslant \rho(\tilde{0}, \tilde{x}) \leqslant m\right\} .
\end{aligned}
$$


Функция $f \in P_{n}$, для которой $N_{f}=S_{m-k, m}^{n}$, называется поясковой булевой функцией.

Используемые, но не определяемые в этой статье понятия и определения можно найти в $[1,2,4]$. Через $\lfloor x\rfloor$ (соответственно $\lceil x\rceil$ ) будем обозначать целую часть (соответственно верхнюю целую часть) числа $x$. Под $\log$ всюду понимается логарифм по основанию 2 . Через $c_{\varepsilon}, c_{\varepsilon}^{\prime}, c_{\varepsilon}^{\prime \prime}$ всюду обозначаются сколь угодно малые положительные константы.

Пусть $\tau(f)$ - число тупиковых д.н.ф. функции $f$, а $\tau(n)$ - максимальное по множеству $P_{n}$ значение этого параметра. Верхняя оценка $\tau(n)$ получается из мощностных соображений (см. с. 125 в [2]). Так как число импликант в тупиковой д.н.ф. не превосходит $2^{n}$, то $\tau(n)$ не превосходит число способов выбрать не более $2^{n}$ из всевозможных $3^{n}$ импликант от $n$ переменных. Поэтому при $n \rightarrow \infty$

$$
\tau(n) \leqslant \sum_{i=0}^{2^{n}}\left(\begin{array}{c}
3^{n} \\
i
\end{array}\right) \leqslant\left(2^{2^{n}}\right)^{c n(1+o(1))},
$$

где $c=\log (3 / 2)$.

Нижние оценки $\tau(n)$ последовательно улучшались в работах ряда авторов. Первый результат был получен С. В. Яблонским (см. с. 123 в [2]) для поясковой функции $N_{f}=S_{m, n-m}^{n}$ :

$$
\tau(n) \geqslant \tau(f) \geqslant(n-1) ! .
$$

Таким образом, было показано, что $\tau(n)$ растет с ростом $n$ значительно быстрее, чем $2^{n}$. Ю. И. Журавлевым [5] было показано, что

$$
\tau(n) \geqslant\left(2^{2^{n}}\right)^{c}, \quad 0<c<1 .
$$

В работе [6] Ю. Л. Васильевым впервые было показано, что значение $\tau(n)$ может превосходить число функций от $n$ переменных, а именно,

$$
\log \tau(n) \gtrsim c 2^{n} \log n, \quad c=1 / 3,
$$

при $n \rightarrow \infty$. А. А. Сапоженко [7] получил асимптотическую оценку $\log \tau(f)$ для почти всех функций $f \in P_{n}$, из которой следовало, что

$$
\log \tau(n) \gtrsim \log n \cdot \log \log n \cdot 2^{n-1} .
$$

Ю. Л. Васильевым (см. с. 126 в [2]) было доказано, что при $n \rightarrow \infty$

$$
\log \tau(n) \gtrsim c \sqrt{n} 2^{n}, \quad c=1 / \sqrt{32 \pi} .
$$

А. А. Сапоженко [8] показал, что такая нижняя оценка с константой $c=\sqrt{2 / \pi}$ достигается на симметрической функции

$$
N_{f}=S_{m-k-1, m-1}^{n} \vee S_{m+1, m+1+k}^{n},
$$

где

$$
m=\lfloor n / 2\rfloor, \quad k=\lfloor n / 4\rfloor .
$$

Исследование симметрических функций и, в частности, поясковых функций (см. [9]) объяснялось фактом существования гипотезы о достижимости значения $\tau(n)$ на классе симметрических функций. Однако существенного улучшения нижней оценки $\tau(n)$ удалось добиться после отказа от исследования конкретных функций и перехода к построению множеств тупиковых д.н.ф. от $n$ переменных. 
Обозначим

$$
T(n)=\sum_{f \in P_{n}} \tau(f)
$$

число тупиковых д.н.ф. от $n$ переменных или число тупиковых комплексов граней в $B^{n}$. Из уже имеющейся нижней оценки $\tau(n)$ вытекает, что

$$
2^{n}=o(\log T(n))
$$

при $n \rightarrow \infty$. Поэтому из очевидного соотношения

$$
\tau(n) \leqslant T(n) \leqslant \tau(n) 2^{2^{n}}
$$

следует, что

$$
\log \tau(n) \sim \log T(n)
$$

при $n \rightarrow \infty$.

В [10] был предложен метод построения множества тупиковых д.н.ф. от $n$ переменных, из которого следует оценка

$$
\log \tau(n) \geqslant c n 2^{n}, \quad c=0,75 \cdot 2^{-5},
$$

при $n \rightarrow \infty$, то есть был получен порядок роста $\log \tau(n)$.

Нижняя оценка для числа тупиковых комплексов граней, по порядку логарифма равная $n 2^{n}$, может быть достигнута только при существовании тупикового комплекса граней, в котором $c_{1} 2^{n}$ граней имеют размерность не менее $c_{2} n$, где $c_{1}, c_{2}$ - положительные константы. Действительно, двоичный догарифм мощности подмножества комплексов граней в кубе $B^{n}$ есть $o\left(n 2^{n}\right)$ при условии, что все грани имеют собственные вершины и либо размерность всех граней есть $o(n)$, либо число граней в комплексе есть $o\left(2^{n}\right)$.

Для параметра

$$
l_{T}(n)=\max _{f \in P_{n}} l_{T}(f),
$$

где $l_{T}(f)-$ наибольшая из длин тупиковых д.н.ф. функции $f \in P_{n}$, имеется очевидная оценка $l_{T}(n)<2^{n}$, так как множество собственных вершин $C_{T}$ не может совпадать с кубом $B^{n}$. Казалось правдоподобным, что $l_{T}(n) \leqslant 2^{n-1}$ и, следовательно, $l_{T}(n)=2^{n-1}$, так как при этом максимум $l_{T}(f)$ достигается на линейной функции $x_{1} \oplus \ldots \oplus x_{n} \bmod 2$ и совпадает с максимальной длиной кратчайшей д.н.ф. Однако С. В. Яблонским [11] был построен пример функции $f \in P_{7}$, для которой $l_{T}(f)=70>2^{7-1}$. В. В. Глаголевым [12] была построена последовательность симметрических функций от $n$ переменных, для которых максимальная длина тупиковой д.н.ф. асимптотически равна $2^{n}$, то есть $l_{T}(n) \sim 2^{n}$ при $n \rightarrow \infty$. Доказательство этой оценки основывается на утверждении о максимальной длине тупиковой д.н.ф. узкой поясковой функции. Для поясковой функции $f \in P_{n}$, где $N_{f}=S_{i, i+k}^{n},(2 k)^{2 k+2}<\ln n$ и $n / 4 \leqslant i<i+k \leqslant 3 n / 4$, существует тупиковая д.н.ф., длина которой асимптотически равна $\left|N_{f}\right|$ при $n \rightarrow \infty$. Тогда для симметрической функции $F=\bigvee_{j=0}^{m} f_{j}$, где $N_{f_{j}}=S_{m_{j}, m_{j}+k}^{n},(2 k)^{2 k+2}<\ln n, m_{j}=\lceil n / 4\rceil+j(k+2)$ и $m=\lfloor n /(2 k+4)\rfloor-1$, компонентами связности являются узкие поясковые функции (см. с. 117 в [2]). Поэтому

$$
l_{T}(F)=\sum_{j=0}^{m} l_{T}\left(f_{j}\right) \sim \sum_{j=0}^{m}\left|N_{f_{j}}\right|=\left|N_{F}\right| \sim 2^{n},
$$


если $k \rightarrow \infty$ и $n \rightarrow \infty$.

Условие $(2 k)^{2 k+2}<\ln n$ или $(2 k+2) \ln (2 k)<\ln \ln n$ выполняется при ширине пояса $\left.\left.k \leqslant 1 / 2-\varepsilon_{n}\right) \ln \ln n /(\ln \ln \ln n\rfloor-1\right)$, где $\varepsilon_{n} \geqslant \ln \ln \ln n / \ln \ln n$. При этом для любой вершины двоичный логарифм мощности пучки граней в таких узких поясах есть $o(\log n \cdot \log \log n)$. Мощность пучков граней не менее $2^{c_{2} n}$ для вершин пояса куба достигается только при ширине пояса $k=c_{1} n$. Однако для длины тупиковой д.н.ф. поясковой функций при ширине пояса $k=c_{1} n$ не известны нижние оценки, которые имеют порядок роста больше $2^{n} / \sqrt{n}$.

В данной статье, на основе развития идей статьи [10] и с использованием вероятностного подхода, усовершенствованы методы построения и оценивания характеристик тупиковых комплексов граней в единичном кубе $B^{n}$, что позволило улучшить оценки экстремальных значений их характеристик. При этом улучшение оценок достигнуто как за счет улучшения оценки числа граней тупикового комплекса, так и за счет увеличения размерности граней, для которой справедливы полученные оценки. В статье приводятся полностью доказательства всех утверждений. Использование геометрической интерпретации делает более наглядной комбинаторные свойства тупиковых комплексов граней в единичном кубе, существенно упрощает и облегчает описание построения структур с экстремальными свойствами, описание которых в аналитической форме, то есть в терминах д.н.ф., представляется весьма затруднительным.

Статья имеет следующую структуру.

В разделе 2 приводится описание методов построения в поясе единичного куба тупиковых комплексов граней, для которых размерность граней совпадает с шириной пояса куба.

В разделе 3 приводятся формулировки основных результатов о числе граней в тупиковом комплексе и числе тупиковых комплексов граней в единичном кубе.

В разделе 4 приводятся доказательства утверждений о максимальном числе граней определенной размерности в тупиковом комплексе.

В разделе 5 приводятся доказательства утверждений о числе тупиковых комплексов граней.

\section{2. Описание конструкции}

Для произвольных вершин $\tilde{\alpha}, \tilde{\beta} \in B^{n}$ будем обозначать $I(\tilde{\alpha}, \tilde{\beta})$ наименьший интервал в $B^{n}$, содержащий одновременно вершины $\tilde{\alpha}$ и $\tilde{\beta}$.

Если для вершин $\tilde{\alpha}=\left(\alpha_{1}, \ldots, \alpha_{n}\right)$ и $\tilde{\beta}=\left(\beta_{1}, \ldots, \beta_{n}\right)$ ввести обозначения

$$
\begin{aligned}
& \tilde{\alpha} \& \tilde{\beta}=\left(\alpha_{1} \& \beta_{1}, \ldots, \alpha_{n} \& \beta_{n}\right), \\
& \tilde{\alpha} \vee \tilde{\beta}=\left(\alpha_{1} \vee \beta_{1}, \ldots, \alpha_{n} \vee \beta_{n}\right),
\end{aligned}
$$

TO

$$
\tilde{\alpha} \& \tilde{\beta} \leqslant \tilde{\alpha} \vee \tilde{\beta}, \quad\|\tilde{\alpha} \vee \tilde{\beta}\|-\|\tilde{\alpha} \& \tilde{\beta}\|=\rho(\tilde{\alpha}, \tilde{\beta}) .
$$

Тогда интервал $I(\tilde{\alpha} \& \tilde{\beta}, \tilde{\alpha} \vee \tilde{\beta})$, то есть интервал с минимальной вершиной $\tilde{\alpha} \& \tilde{\beta}$ и максимальной вершиной $\tilde{\alpha} \vee \tilde{\beta}$, является минимальным интервалом, который содержит одновременно вершины $\tilde{\alpha}$ и $\tilde{\beta}$ и имеет размерность $\rho(\tilde{\alpha}, \tilde{\beta})$. Множество вершин $\left\{\tilde{x} \in B^{n}: \rho(\tilde{\alpha}, \tilde{x})+\rho(\tilde{x}, \tilde{\beta}) \leqslant \rho(\tilde{\alpha}, \tilde{\beta})\right\}$ в случае сравнимых вершин $\tilde{\alpha} \leqslant \tilde{\beta}$ совпадает с интервалом $I(\tilde{\alpha}, \tilde{\beta})$, а в случае несравнимых вершин $\tilde{\alpha}$ и $\tilde{\beta}$ совпадает с интервалом 
$I(\tilde{\alpha} \& \tilde{\beta}, \tilde{\alpha} \vee \tilde{\beta})$, то есть представляет наименьший интервал, содержащий одновременно вершины $\tilde{\alpha}$ и $\tilde{\beta}$.

Определение 1. Пусть $S$ - произвольное подмножество вершин $B^{n}$ и $A-$ подмножество $S$.

Вершина $\tilde{x} \in S$ называется $(A, S)$-внутренней вершиной, если существует вершина $\tilde{\alpha} \in A$ такая, что $I(\tilde{\alpha}, \tilde{x}) \subseteq S$, и при этом интервал $I(\tilde{\alpha}, \tilde{x})$ не является максимальным для подмножества $S$.

Вершина $\tilde{x} \in S$ называется $(A, S)$-граничной вершиной, если для любой вершины $\tilde{\alpha} \in A$, либо $I(\tilde{\alpha}, \tilde{x}) \subseteq S$ и интервал $I(\tilde{\alpha}, \tilde{x})$ является максимальным для подмножества $S$, либо $I(\tilde{\alpha}, \tilde{x}) \nsubseteq S$.

Подмножество $A \subset S$, относительно которого рассматриваются множества граничных и внутренних вершин, будем называть опорным подмножеством. Множество $(A, S)$-внутренних вершин будем обозначать через $W(A, S)$, а множество $(A, S)$-граничных вершин - через $G(A, S)$.

Лемма 1. Пусть $A \subset S$ и для $l=|G(A, S)|$ комплекс интервалов

$$
I(A, S)=\left\{I_{j}=I\left(\tilde{x}_{j}, \tilde{\alpha}\right), j=1, \ldots, l\right\}
$$

определяется следуюшим образом: для каждой $(A, S)$-граничной вершины $\tilde{x}_{j}, j=1, \ldots, l$, в комплекс включается один интервал, который содержит вериину $\tilde{x}_{j}$ и некоторую вершину $\tilde{\alpha} \in A$, для которой интервал $I\left(\tilde{x}_{j}, \tilde{\alpha}\right) S$ принадлежит $S$ и является максимальным для подмножества $S$. Тогда комплекс интервалов $I(A, S)$ является тупиковым, при этом каждая вершина $\tilde{x}_{j} \in G(A, S)$ является собственной вершиной для покрывающего ее интервала $I\left(\tilde{x}_{j}, \tilde{\alpha}\right) \in I(A, S)$.

Доказательство. Действительно, во-первых,

$$
I\left(\tilde{x}_{j}, \tilde{\alpha}\right) \cap A=\{\tilde{\alpha}\},
$$

то есть в интервале $I\left(\tilde{x}_{j}, \tilde{\alpha}\right)$ нет других вершин из $A$. Если $\tilde{\alpha}_{1} \neq \tilde{\alpha}$ и $\tilde{\alpha}_{1} \in I\left(\tilde{x}_{j}, \tilde{\alpha}\right)$, то

$$
I\left(\tilde{x}_{j}, \tilde{\alpha}_{1}\right) \subset I\left(\tilde{x}_{j}, \tilde{\alpha}\right) \subseteq S
$$

то есть $I\left(\tilde{x}_{j}, \tilde{\alpha}_{1}\right)$ содержится в $S$ и не является максимальным в $S$. Поэтому из включения $\tilde{\alpha}_{1} \in A$, следует что $\tilde{x}_{j} \in W(A, S)$, то есть не может быть $(A, S)$-граничной вершиной.

Во-вторых,

$$
I\left(\tilde{x}_{j}, \tilde{\alpha}\right) \cap G(A, S)=\left\{\tilde{x}_{j}\right\}
$$

так как для остальных вершин $\tilde{x} \in I\left(\tilde{x}_{j}, \tilde{\alpha}\right)$ при $\tilde{x} \neq \tilde{x}_{j}$ интервал $I(\tilde{x}, \tilde{\alpha}) \subset I\left(\tilde{x}_{j}, \tilde{\alpha}\right)$, то есть не является максимальным в $S$, соответственно такие вершины по определению принадлежат $W(A, S)$.

Каждый интервал $I\left(\tilde{x}_{j}, \tilde{\alpha}\right) \in I(A, S)$ является максимальным для множества вершин покрываемых комплексом $I(A, S)$, так как является максимальным для множества $S$. При этом он содержит ровно одну вершину из опорного множества $A$ и ровно одну $(A, S)$-граничную вершину, то есть вершина $\tilde{x}_{j}$ является собственной для интервала $I\left(\tilde{x}_{j}, \tilde{\alpha}\right)$ в комплексе интервалов $I(A, S)$.

Лемма доказана. 


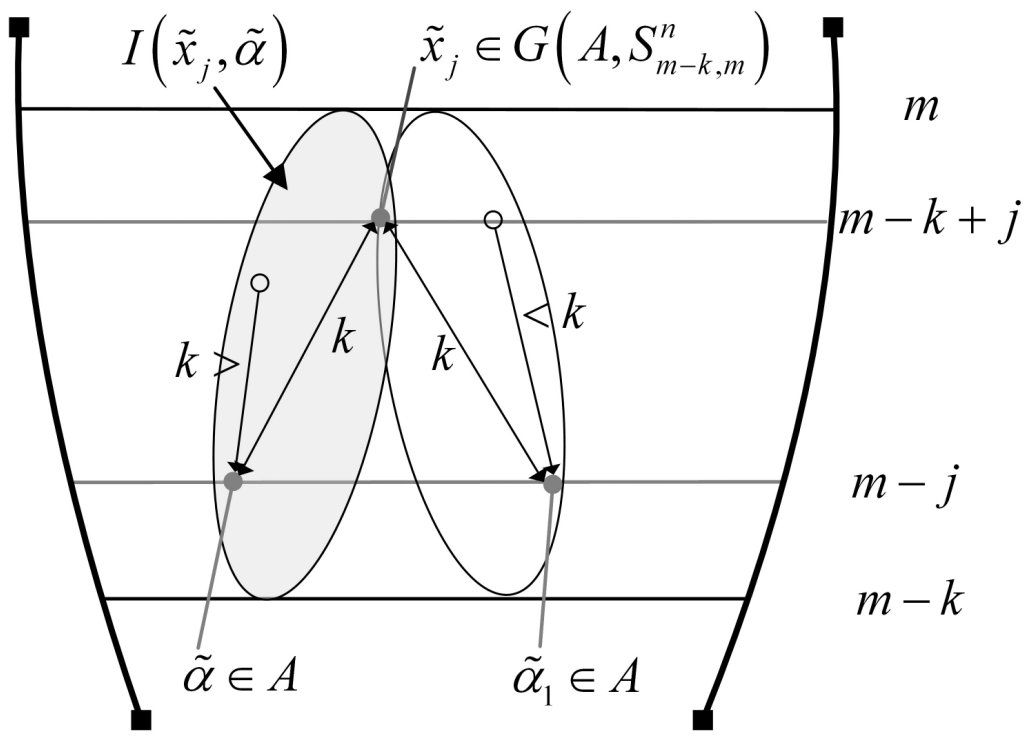

Рис. 1. Внутренние и граничные вершины для $A \subset S_{m-k, m}^{n}$

В качестве подмножества $S \subset B^{n}$ будем рассматривать пояс

$$
S=S_{m-k, m}^{n}=\bigcup_{i=m-k}^{m} B_{i}^{n}
$$

в единичном кубе, состоящий из слоев с номерами $i=m-k, \ldots, m$. Любой максимальный интервал для $S_{m-k, m}^{n}$ имеет размерность $k$. Поэтому для любого интервала $I=I\left(\tilde{x}_{j}, \tilde{\alpha}\right) \in I\left(A, S_{m-k, m}^{n}\right)$, который является максимальным в поясе $S_{m-k, m}^{n}$ и минимальным интервалом, содержащим вершины $\tilde{x}_{j}$ и $\tilde{\alpha}$, справедливо равенство

$$
\rho\left(\tilde{x}_{j}, \tilde{\alpha}\right)=k .
$$

Определение $(A, S)$-внутренних и граничных вершин для подмножества $A \subset S=$ $S_{m-k, m}^{n}$ эквивалентно следующему определению (см. рис. 1).

Вершина $\tilde{x} \in S_{m-k, m}^{n}$ называется $\left(A, S_{m-k, m}^{n}\right)$-внутренней вершиной, если существует вершина $\tilde{\alpha} \in A$ такая, что $I(\tilde{\alpha}, \tilde{x}) \subset S_{m-k, m}^{n}$ и $\rho(\tilde{\alpha}, \tilde{x})<k$.

Вершина $\tilde{x} \in S_{m-k, m}^{n}$ называется $\left(A, S_{m-k, m}^{n}\right)$-граничной вершиной, если она не является $\left(A, S_{m-k, m}^{n}\right)$-внутренней вершиной и существует вершина $\tilde{\alpha} \in A$ такая, что $I(\tilde{\alpha}, \tilde{x}) \subset S_{m-k, m}^{n}$ и $\rho(\tilde{\alpha}, \tilde{x})=k$.

Конструкция, используемая для построения экстремальных тупиковых комплексов из интервалов заданной размерности в поясе куба, основывается на лемме 1 . Комплекс интервалов $I\left(A, S_{m-k, m}^{n}\right)$, построенный по множеству $\left(A, S_{m-k, m}^{n}\right)$-граничных вершин для опорного множества $A$, определяет тупиковый комплекс $k$-мерных интервалов. При этом число интервалов в комплексе равно $\left|G\left(A, S_{m-k, m}^{n}\right)\right|$ и все $\left(A, S_{m-k, m}^{n}\right)$-граничные вершины являются собственными для покрывающих их интервалов. Соответственно задача 
построения тупикового комплекса из $k$-мерных интервалов в $S_{m-k, m}^{n}$, число которых сравнимо с мощностью пояса, сводится к задаче построения опорного множества $A \subset S_{m-k, m}^{n}$, для которого число $\left(A, S_{m-k, m}^{n}\right)$-граничных вершин сравнимо с мощностью пояса.

Комплекс интервалов $I\left(A, S_{m-k, m}^{n}\right)$ определяется неоднозначно, если для вершины $\tilde{x} \in G\left(A, S_{m-k, m}^{n}\right)$ существуют несколько различных вершин опорного множества $\tilde{\alpha}_{i} \in A$, для которых интервал $I\left(\tilde{\alpha}_{i}, \tilde{x}\right) \subset S_{m-k, m}^{n}$ имеет размерность $k$. Поэтому будем обозначать $T\left(S_{m-k, m}^{n}, A\right)$ множество тупиковых комплексов $k$-мерных интервалов в $S_{m-k, m}^{n}$, которые могут быть построены по множеству $\left(A, S_{m-k, m}^{n}\right)$-граничных вершин опорного множества $A, T_{m-k, m}^{n}$ множество произвольных тупиковых комплексов $k$-мерных интервалов в $S_{m-k, m}^{n}$.

Конструкция, используемая для построения множества тупиковых комплексов по произвольному тупиковому комплексу $T \in T_{m-k_{0}, m}^{n}$, основана на сечении по слою $B_{m-k}^{n}$ интервалов тупикового комплекса, имеющих собственные вершины в поясе $S_{m-p, m}^{n}$. При этом параметры $p$ и $k$ должны удовлетворять соотношению $0<p<k<k_{0}$. В результате такого сечения в поясе $S_{m-k, m}^{n}$ получим пучки интервалов размерности $k$, которые имеют мощность не менее $\left(\begin{array}{c}k_{0}-p \\ k-p\end{array}\right)$. Возьмем для каждой вершины из $C_{T} \cap S_{m-p, m}^{n}$ по одному интервалу из пучка $k$-мерных интервалов и получим не менее $\left(\begin{array}{c}k_{0}-p \\ k-p\end{array}\right)^{\left|C_{T} \cap S_{m-p, m}^{n}\right|}$ различных комплексов интервалов, которые являются тупиковыми комплексами из $T_{m-k, m}^{n}$. При $c_{1} n \leqslant m \leqslant n / 2$ и $k_{0}=c_{2} n$ выбор параметров $p=o(n), p / \sqrt{n} \rightarrow \infty$ и $k=\left\lfloor c k_{0}\right\rfloor$, где $0<c<1$, дает оценку

$$
\log \left|T_{m-k, m}^{n}\right| \gtrsim k_{0}\left|C_{T}\right| H(c)
$$

при $n \rightarrow \infty$.

Существование тупиковых комплексов, в которые входят порядка $2^{n}$ интервалов размерности порядка $n$ является необходимым условием для возможности нижней оценки логарифма числа тупиковых комплексов порядка $n 2^{n}$. Это вытекает из следующего утверждения.

Предложение 1. Пусть $D_{n}(l, k)$ - множество комплексов интервалов в единичном кубе $B^{n}$, для которых число интервалов в комплексе не более $l$, все интерваль имеют размерность не более $k$ и имеют собственнье вершины. Если $l=o\left(2^{n}\right)$ или $k=o(n)$ при $n \rightarrow \infty$, mo

$$
\log _{2}\left|D_{n}(l, k)\right|=o\left(n 2^{n}\right) .
$$

Доказательство. Для вершины $\tilde{\alpha}$ мощность пучка интервалов размерности не более $k$ не превосходит

$$
s_{k}^{n}=\left|S_{0, k}^{n}\right|=\sum_{i=0}^{k}\left(\begin{array}{l}
n \\
i
\end{array}\right),
$$

а число способов выбрать в $B^{n}$ подмножество собственных вершин мощности $j$ не превосходит $\left(\begin{array}{c}2^{n} \\ j\end{array}\right)$. Тогда

$$
\left|D_{n}(l, k)\right| \leqslant \sum_{j=0}^{l}\left(s_{k}^{n}\right)^{j}\left(\begin{array}{c}
2^{n} \\
j
\end{array}\right) \leqslant\left(s_{k}^{n}\right)^{l} \sum_{j=0}^{l}\left(\begin{array}{c}
2^{n} \\
j
\end{array}\right) \leqslant 2^{2^{n}}\left(s_{k}^{n}\right)^{l} .
$$

При $l(n)=o\left(2^{n}\right)$, используя оценку $s_{k}^{n} \leqslant 2^{n}$, получим равенство

$$
\log _{2} 2^{2^{n}}\left(s_{k}^{n}\right)^{l(n)}=o\left(n 2^{n}\right) .
$$


При $k=o(n)$ используем оценку

$$
s_{k}^{n}=\sum_{i=0}^{k}\left(\begin{array}{l}
n \\
i
\end{array}\right) \leqslant 2^{n H(k / n)},
$$

справедливую для $k / n<1 / 2$, где

$$
H(x)=-x \log x-(1-x) \log (1-x)
$$

(см. с. 280 в [13]). Так как $k=o(n)$, видим, что

$$
H(k / n) \sim(k / n) \log (n / k)=o(1)
$$

при $n \rightarrow \infty$, и тогда

$$
\log _{2}\left(2^{2^{n}}\left(s_{k}^{n}\right)^{l(n)}\right) \leqslant \log _{2}\left(2^{2^{n}} 2^{2^{n} n H(k / n)}\right)=o\left(n 2^{n}\right) .
$$

Предложение доказано.

Таким образом, для получения высоких оценок числа тупиковых комплексов граней на основе предлагаемого подхода, ключевой проблемой является построение опорного множества вершин в поясе единичного куба, при этом ширина пояса должна быть порядка $n$, число вершин в поясе должно быть порядка $2^{n}$ и число граничных вершин должно быть сравнимо с мощностью пояса.

\section{3. Формулировка основных результатов}

Доказано, что для $c_{\varepsilon}^{\prime} n \leqslant m \leqslant n / 2$ и $k \leqslant m\left(1-c_{\varepsilon}^{\prime \prime}\right) / 2$ существует опорное подмножество $A_{\lambda}$, мощность которого определяется параметром $\lambda$, где $c_{\varepsilon}<\lambda<m / k-2-c_{\varepsilon}$ и $\lambda=o(n)$ при $n \rightarrow \infty$. Для числа граничных вершин при $n \rightarrow \infty$ справедлива оценка

$$
\left|S_{m-r, m}^{n}\right|\left(1-e^{-\lambda}\right)\left(1-\frac{\lambda k}{m-2 k}\right) \lesssim\left|G\left(A_{\lambda}, S_{m-k, m}^{n}\right)\right| .
$$

С использованием лемм 1-5, которые будут доказаны ниже, для построения тупикового комплекса интервалов в поясе куба доказывается следующая теорема.

Теорема 1. Пусть $S=S_{m-k, m}^{n} u m_{S}=m$ или $S=S_{n-m, n-m+k}^{n} u m_{S}=n-m$, где $c_{\varepsilon}^{\prime} n \leqslant m \leqslant n / 2 u k \leqslant\left(m_{S} / 2\right)\left(1-c_{\varepsilon}^{\prime \prime}\right)$. Существует опорное множество $A_{\lambda} \subset S$ такое, что для тупикового комплекса интервалов $T \in T\left(S, A_{\lambda}\right)$ при $n \rightarrow \infty$

$$
|S|\left(1-e^{-\lambda}\right)\left(1-\frac{\lambda k}{m_{S}-2 k}\right) \lesssim l(T)=\left|G\left(A_{\lambda}, S\right)\right| \leqslant|S|,
$$

где $c_{\varepsilon}<\lambda<m_{S} / k-2-c_{\varepsilon} u \lambda=o(n)$ при $n \rightarrow \infty$. При этом в тупиковом комплексе $T$ каждый интервал имеет размерность $k$, содержит одну вершину из опорного подмножества $A_{\lambda}$ и одну вершину из множества $\left(A_{\lambda}, S\right)$-граничных вершин, которая является собственной для покрывающего ее интервала.

В качестве следствия из теоремы 1 получаем следующие два утверждения. 
(1) Если $k=o(n)$, то полагая $\lambda=\ln \left(m_{S} / k\right)=o(n)$, получаем, что

$$
l(T) \sim|S|
$$

при $n \rightarrow \infty$.

(2) Если $c_{\varepsilon}^{\prime} n \leqslant k \leqslant\left(m_{S} / 2\right)\left(1-c_{\varepsilon}^{\prime \prime}\right)$, то

$$
l(T) \gtrsim|S| \varphi\left(\lambda_{0}, x\right)
$$

при $n \rightarrow \infty$, где $x=k / m_{S}$ и

$$
\varphi(\lambda, x)=\left(1-e^{-\lambda}\right)\left(1-\frac{\lambda x}{1-2 x}\right) .
$$

Значение $\lambda_{0}$ выбирается из условия максимизации по $\lambda$ функции $\varphi(\lambda, x)$ при $0<\lambda<1 / x-2$ и $\varphi\left(\lambda_{0}, x\right)$ удовлетворяет соотношению $0<\varphi\left(\lambda_{0}, x\right)<1$.

Для максимального числа $k$-мерных интервалов $l_{T}(n, k)$ в тупиковом комплексе получены следующие оценки: $l_{T}(n, k) \sim 2^{n}$, если $k=o(n), k \rightarrow \infty$, и $l_{T}(n, k) \geqslant 2^{n}$, если $c_{\varepsilon}^{\prime} n \leqslant k \leqslant(n / 4)\left(1-c_{\varepsilon}^{\prime \prime}\right)$ и $n \rightarrow \infty$. Также доказано (см. теорему 2), что при $k \geqslant c(n+1)$ число интервалов размерности не менее $k$ в тупиковом комплексе не превосходит $(1-c / 2) 2^{n}$.

Нижние оценки числа тупиковых комплексов интервалов получены путем построения по тупиковому комплексу $T \in T_{m-k_{0}, m}^{n}$ множества тупиковых комплексов $\Omega_{\tau}^{n}\left(T, C_{T}, S_{m-k_{0}, m}^{n}, p, k\right) \subset T_{m-k, m}^{n}$, где параметры $p$ и $k$ удовлетворяют условию $0<p<k<k_{0}$. С использованием оценки максимального числа интервалов в тупиковом комплексе доказывается, что если $c_{\varepsilon} n \leqslant m \leqslant n / 2, c_{\varepsilon}^{\prime} n \leqslant k_{0} \leqslant(m / 2)\left(1-c_{\varepsilon}^{\prime \prime}\right)$, $k_{0} / m \sim x, k<k_{0}$ и $k / \sqrt{n} \rightarrow \infty$ при $n \rightarrow \infty$, то (см. теорему 3 )

$$
\begin{aligned}
\log \left|T_{m-k, m}^{n}\right| & =\log \left|T_{n-m, n-m+k}^{n}\right| \\
& \gtrsim\left|S_{m-k, m}^{n}\right| k_{0} H\left(k / k_{0}\right) \varphi(\lambda, x) .
\end{aligned}
$$

Как следствие, отсюда вытекает, что

$$
\log \left|T_{m-k, m}^{n}\right|=\log \left|T_{n-m, n-m+k}^{n}\right| \asymp n 2^{n}
$$

при $n \rightarrow \infty$, если $\lfloor n / 2\rfloor-c_{1} / \sqrt{n} \leqslant m \leqslant\lfloor n / 2\rfloor$, где $c_{1}-$ произвольная постоянная, и $k \sim x m$, где $0<x<0,5$, то есть для логарифма числа тупиковых комплексов $k$-мерных интервалов в кубе $B^{n}$ получен порядок роста $n 2^{n}$ при $n \rightarrow \infty$, если $k \sim c n$ и $0<c<1 / 4$.

При оптимальном выборе параметров для числа тупиковых комплексов в $B^{n}$ и для максимального числа тупиковых д.н.ф. функции из $P_{n}$ в силу того, что $\log \tau(n) \sim \log T(n)$, получена оценка (см. теорему 4)

$$
\begin{aligned}
\log T(n) & \geqslant \log \left|T_{m-1-k, m-1}^{n}\right|+\log \left|T_{n-m+1, n-m+1+k}^{n}\right| \\
& >1,355 n 2^{n-5},
\end{aligned}
$$

где $m=\lfloor n / 2\rfloor$ и $k \approx 0,0526 n$ при $n \rightarrow \infty$. 


\section{4. Оценки числа граней тупиковых комплексов}

Докажем вспомогательные леммы 2 и 3 о свойствах и количественных соотношениях для внутренних и граничных вершин в поясе $S_{m-k, m}^{n}$ для опорного множества, состоящего из одной вершины $A=\{\tilde{\alpha}\}$. Далее будем обозначать $W(\tilde{\alpha})=W\left(\{\tilde{\alpha}\}, S_{m-k, m}^{n}\right)$ и $G(\tilde{\alpha})=G\left(\{\tilde{\alpha}\}, S_{m-k, m}^{n}\right)$ множества внутренних и граничных вершин, соответственно, $w(\tilde{\alpha})=|W(\tilde{\alpha})|=w_{m-j}$ и $g(\tilde{\alpha})=|G(\tilde{\alpha})|=w_{m-j}-$ число внутренних и граничных вершин для $\tilde{\alpha} \in B_{m-j}^{n}$.

Лемма 2. Пусть $\tilde{\alpha} \in B_{m-j}^{n}$, где $0 \leqslant j \leqslant k$. Тогда

$$
w_{m-j}=\left(\begin{array}{c}
n \\
m-j
\end{array}\right)^{-1} \sum_{s=0}^{k-1}\left(\begin{array}{l}
n \\
s
\end{array}\right) \sum_{t=\max \{0, s-j\}}^{\min \{k-j, s\}}\left(\begin{array}{l}
s \\
t
\end{array}\right)\left(\begin{array}{c}
n-s \\
m-j-t
\end{array}\right),
$$

справедливо включение $G(\tilde{\alpha}) \subset B_{m-k+j}^{n} u$

$$
\begin{aligned}
g_{m-j} & =\left(\begin{array}{l}
m-j \\
k-j
\end{array}\right)\left(\begin{array}{c}
n-m+j \\
j
\end{array}\right)=\left(\begin{array}{l}
m-j \\
m-k
\end{array}\right)\left(\begin{array}{c}
n-m+j \\
n-m
\end{array}\right) \\
& =\left(\begin{array}{l}
n \\
k
\end{array}\right)\left(\begin{array}{c}
k \\
k-j
\end{array}\right)\left(\begin{array}{l}
n-k \\
m-k
\end{array}\right) /\left(\begin{array}{c}
n \\
m-j
\end{array}\right) .
\end{aligned}
$$

Доказательство. Проведем подсчет числа вершин $\tilde{\beta}$, для которых $\rho(\tilde{\alpha}, \tilde{\beta})=s$ и $I(\tilde{\alpha}, \tilde{\beta}) \subset S_{m-k, m}^{n}$, где $0 \leqslant s \leqslant k$. Заметим, что условие $I(\tilde{\alpha}, \tilde{\beta}) \subset S_{m-k, m}^{n}$ эквивалентно условиям $\|\tilde{\alpha} \& \tilde{\beta}\| \geqslant m-k$ и $l\|\tilde{\alpha} \vee \tilde{\beta}\| \leqslant m$. Пусть вершины $\tilde{\alpha}, \tilde{\beta}, \tilde{\alpha} \vee \tilde{\beta}$ и $\tilde{\alpha} \& \tilde{\beta}$ имеют следующий вид:

$$
\begin{array}{rlrl}
\tilde{\alpha} & =(\underbrace{1 \ldots 1}_{m-j} 0 \ldots 0), & \|\tilde{\alpha}\| & =m-j, \\
\tilde{\beta} & =(\underbrace{1 \ldots 1}_{m-j-t}, \underbrace{0 \ldots 0}_{t} \underbrace{1 \ldots 1}_{s-t} 0 \ldots 0), & \rho(\tilde{\alpha}, \tilde{\beta})=s, \quad t \leqslant s, \\
\tilde{\alpha} \vee \tilde{\beta} & =(\underbrace{1 \ldots 1}_{m-j+s-t} 0 \ldots 0), & \|\tilde{\alpha} \vee \tilde{\beta}\|=m-j+s-t, \\
\tilde{\alpha} \& \tilde{\beta} & =(\underbrace{1 \ldots 1}_{m-j-t} 0 \ldots 0), & \|\tilde{\alpha} \& \tilde{\beta}\|=m-j-t .
\end{array}
$$

Интервал $I(\tilde{\alpha}, \tilde{\beta}) \subset S_{m-k, m}^{n}$ тогда и только тогда, когда $m-j-t \geqslant m-k$ и $m-j+s-t \leqslant m$, то есть $s-j \leqslant t \leqslant k-j$. Таким образом, число таких вершин $\tilde{\beta}$ равно

$$
\sum_{t=\max \{0, s-j\}}^{\min \{s, k-j\}}\left(\begin{array}{c}
m-j \\
t
\end{array}\right)\left(\begin{array}{c}
n-m+j \\
s-t
\end{array}\right)
$$


Тогда

$$
\begin{aligned}
& w(\tilde{\alpha})=\sum_{s=0}^{k-1} \sum_{t=\max \{0, s-j\}}^{\min \{s, k-j\}}\left(\begin{array}{c}
m-j \\
t
\end{array}\right)\left(\begin{array}{c}
n-m+j \\
s-t
\end{array}\right), \\
& g(\tilde{\alpha})=\sum_{t=\max \{0, k-j\}}^{\min \{k, k-j\}}\left(\begin{array}{c}
m-j \\
t
\end{array}\right)\left(\begin{array}{c}
n-m+j \\
k-t
\end{array}\right)=\left(\begin{array}{c}
m-j \\
k-j
\end{array}\right)\left(\begin{array}{c}
n-m+j \\
j
\end{array}\right) .
\end{aligned}
$$

При этом, если $\tilde{\beta} \in G(\tilde{\alpha})$, то индекс $t$ принимает единственное значение $t=k-j$ и $\|\tilde{\beta}\|=m-k+j$, то есть $G(\tilde{\alpha}) \subset B_{m-k+j}^{n}$. Заметим также, что при любых допустимых значениях $t, s, j$ имеет место соотношение

$$
\left(\begin{array}{c}
m-j \\
t
\end{array}\right)\left(\begin{array}{c}
n-m+j \\
s-t
\end{array}\right)=\left(\begin{array}{l}
n \\
s
\end{array}\right)\left(\begin{array}{l}
s \\
t
\end{array}\right)\left(\begin{array}{c}
n-s \\
m-j-t
\end{array}\right) /\left(\begin{array}{c}
n \\
m-j
\end{array}\right) .
$$

Отсюда следуют утверждения леммы.

Лемма 3. (1) Последовательность $\left\{g_{m-j}, j=0,1, \ldots, k\right\}$ является унимодальной (см. c. 178 в [4]) и выполняются неравенства

$$
g_{m-k}<g_{m-k+1}<\ldots<g_{m-j_{0}-1} \leqslant g_{m-j_{0}}>g_{m-j_{0}+1}>\ldots>g_{m-1}>g_{m},
$$

где

$$
j_{0}=\left\lfloor\frac{k(n-m+1)-m}{n-k}\right\rfloor=\left\lfloor k-\frac{k(m-k-1)+m}{(n-k)}\right\rfloor .
$$

При этом $g_{m} \leqslant g_{m-k}$ для $m \leqslant(n+k) / 2 u g_{m}>g_{m-k}$ для $m>(n+k) / 2$.

(2) Выполняется равенство

$$
\left(\begin{array}{c}
n \\
m-k+j
\end{array}\right) g_{m-k+j}=\left(\begin{array}{c}
n \\
m-j
\end{array}\right) g_{m-j}
$$

(3) Выполняется равенство

$$
\sum_{j=0}^{k} g_{m-j}=\left(\begin{array}{c}
n \\
k+1
\end{array}\right)
$$

(4) Выполняется неравенство

$$
w_{m-j} / g_{m-j} \leqslant\left|S_{0, k-1}^{m}\right| /\left|B_{k}^{m}\right|,
$$

если $m \leqslant n / 2, u$

$$
w_{m-j} / g_{m-j} \leqslant k / m-2 k,
$$

если при этом $k \leqslant m\left(1-c_{\varepsilon}\right) / 2$, для $j=0,1 \ldots, k$. 
Доказательство. Так как

$$
\frac{g_{m-j}}{g_{m-(j+1)}}=\frac{(m-j)(j+1)}{(k-j)(n-m+j+1)},
$$

то условие $g_{m-j} / g_{m-(j+1)} \leqslant 1$ верно для $j \leqslant j_{0}=\lfloor(k(n-m+1)-m) /(n-k)\rfloor$ и может быть $g_{m-\left(j_{0}+1\right)}=g_{m-j_{0}}$, а для $j>j_{0}+1$ выполняется неравенство $g_{m-j} / g_{m-(j+1)}>1$, то есть выполняются неравенства

$$
g_{m-k}<g_{m-k+1}<\ldots<g_{m-j_{0}-1} \leqslant g_{m-j_{0}}>g_{m-j_{0}+1}>\ldots>g_{m-1}>g_{m} .
$$

При этом для $m \leqslant n-m+k$, то есть при $m \leqslant(n+k) / 2$, выполняется неравенство

$$
g_{m}=\left(\begin{array}{l}
m \\
k
\end{array}\right) \leqslant\left(\begin{array}{c}
n-m+k \\
k
\end{array}\right)=g_{m-k},
$$

и наоборот, $g_{m-k} \leqslant g_{m}$ при $m \geqslant(n+k) / 2$.

Непосредственно подстановкой значений $g_{m-j}$ и $g_{m-k+j}$ проверяется справедливость соотношения

то есть справедливость равенства

$$
\left(\begin{array}{c}
n \\
m-k+j
\end{array}\right) g_{m-k+j}=\left(\begin{array}{c}
n \\
m-j
\end{array}\right) g_{m-j},
$$

$$
\begin{aligned}
\left(\begin{array}{c}
n \\
m-k+j
\end{array}\right)\left(\begin{array}{c}
m-k+j \\
j
\end{array}\right)\left(\begin{array}{c}
n-m+k-j \\
k-j
\end{array}\right) & =\left(\begin{array}{l}
k \\
j
\end{array}\right)\left(\begin{array}{l}
m \\
k
\end{array}\right)\left(\begin{array}{l}
n \\
m
\end{array}\right) \\
& =\left(\begin{array}{c}
n \\
m-j
\end{array}\right)\left(\begin{array}{c}
m-j \\
k-j
\end{array}\right)\left(\begin{array}{c}
n-m+j \\
j
\end{array}\right) .
\end{aligned}
$$

Подставляя $a=n-m$ и $b=m-k$ в соотношение

$$
\sum_{j=0}^{k}\left(\begin{array}{c}
a+j \\
a
\end{array}\right)\left(\begin{array}{c}
b+k-j \\
b
\end{array}\right)=\left(\begin{array}{c}
a+b+k+1 \\
k
\end{array}\right)
$$

(см. соотношение (3.10) на с. 268 в [13]) и учитывая, что $a+b+k+1=n+1$, получаем равенство

$$
\sum_{j=0}^{k} g_{m-j}=\sum_{j=0}^{k}\left(\begin{array}{c}
n-m+j \\
n-m
\end{array}\right)\left(\begin{array}{l}
m-j \\
m-k
\end{array}\right)=\left(\begin{array}{c}
n+1 \\
k
\end{array}\right)
$$

Введем обозначение

$$
R(n, m, k, s, j)=\left(\left(\begin{array}{c}
k \\
k-j
\end{array}\right)\left(\begin{array}{c}
n-s \\
m-s
\end{array}\right)\right)^{-1} \sum_{t=\max \{0, s-j\}}^{\min \{s, k-j\}}\left(\begin{array}{l}
s \\
t
\end{array}\right)\left(\begin{array}{c}
n-s \\
m-j-t
\end{array}\right) .
$$

Тогда для отношения $w_{m-j} / g_{m-j}$ получаем представление

$$
\begin{aligned}
\frac{w_{m-j}}{g_{m-j}} & =\sum_{s=0}^{k-1}\left(\left(\begin{array}{l}
n \\
s
\end{array}\right)\left(\begin{array}{c}
n-s \\
m-s
\end{array}\right)\right) /\left(\left(\begin{array}{l}
n \\
k
\end{array}\right)\left(\begin{array}{l}
n-k \\
m-k
\end{array}\right)\right) R(n, m, k, s, j) \\
& =\sum_{s=0}^{k-1}\left(\begin{array}{l}
m \\
s
\end{array}\right) /\left(\begin{array}{l}
m \\
k
\end{array}\right) R(n, m, k, s, j) .
\end{aligned}
$$


При $m \leqslant n / 2$ и $j+t \geqslant s$ выполняется неравенство $m-j-t \leqslant m-s \leqslant(n-s) / 2$. Поэтому имеет место соотношение

$$
\left(\begin{array}{c}
n-s \\
m-j-t
\end{array}\right) \leqslant\left(\begin{array}{c}
n-s \\
m-s
\end{array}\right)
$$

так как последовательность $\left(\begin{array}{c}n-s \\ i\end{array}\right)$, где $i=1, \ldots, n-s$, является унимодальной и возрастает при $i \leqslant(n-s) / 2$. При $0 \leqslant j \leqslant k$ и $0 \leqslant s \leqslant k-1$ справедливо соотношение

$$
\sum_{t=\max \{0, s-j\}}^{\min \{s, k-j\}}\left(\begin{array}{l}
s \\
t
\end{array}\right) \leqslant \sum_{t=\max \{0, s-j\}}^{\min \{s, k-j\}}\left(\begin{array}{l}
s \\
t
\end{array}\right)\left(\begin{array}{c}
k-s \\
k-j-t
\end{array}\right)=\left(\begin{array}{c}
k \\
k-j
\end{array}\right)
$$

(см. соотношение (3.10) на с. 268 в [13]).

Используя приведенные неравенства, получаем оценку $R(n, m, k, s, j) \leqslant 1$, то есть для $m \leqslant n / 2$ доказано, что

$$
\frac{w_{m-j}}{g_{m-j}} \leqslant\left(\begin{array}{c}
m \\
k
\end{array}\right)^{-1} \sum_{s=0}^{k-1}\left(\begin{array}{c}
m \\
s
\end{array}\right)=\frac{\left|S_{0, k-1}^{m}\right|}{\left|B_{k}^{m}\right|} .
$$

При $k \leqslant m\left(1-c_{\varepsilon}\right) / 2$ справедлива оценка

$$
\begin{aligned}
\frac{w_{m-j}}{g_{m-j}} & \leqslant \sum_{s=0}^{k-1}\left(\begin{array}{l}
m \\
s
\end{array}\right)\left(\begin{array}{l}
m \\
k
\end{array}\right)^{-1} \leqslant \sum_{s=0}^{k-1}\left(\frac{k}{m-k}\right)^{k-s} \\
& =\sum_{i=1}^{k} q^{i} \leqslant \frac{q}{1-q}=\frac{k}{m-2 k},
\end{aligned}
$$

так как справедливо соотношение

$$
q=\frac{k}{m-k} \leqslant \frac{1-c_{\varepsilon}}{1+c_{\varepsilon}}<1 .
$$

Лемма доказана.

Далее нам понадобится следующее почти очевидное вероятностное утверждение.

Лемма 4. Пусть $S$ - конечное множество, в котором заданы два непустыле непересекающиеся подмножества $X \subset S, Y \subset S u X \cap Y=\varnothing$. Если подмножество А фиксированной мощчности выбирается случайным образом в $S$ и $|A|+|X|+|Y|<|S|$, то

$$
\mathbf{P}\{(X \cap A \neq \varnothing) \cap(Y \cap A \neq \varnothing)\}<\mathbf{P}\{X \cap A \neq \varnothing\} \mathbf{P}\{Y \cap A \neq \varnothing\} .
$$

Доказательство. Очевидно, что

$$
\begin{aligned}
\mathbf{P}\{(X \cap A \neq \varnothing) \cap(Y \cap A \neq \varnothing)\}= & \mathbf{P}\{Y \cap A=\varnothing\}-\mathbf{P}\{X(\cap A \neq \varnothing) \cap(Y \cap A \neq \varnothing)\} \\
= & \mathbf{P}\{(X \cap A \neq \varnothing)\} \mathbf{P}\left\{Y \cap A^{\prime} \neq \varnothing\right\} \\
= & 1-\mathbf{P}\{Y \cap A=\varnothing\}-\mathbf{P}\{(X \cap A=\varnothing) \cap(Y \cap A=\varnothing\} \\
& -\mathbf{P}\{(X \cap A=\varnothing) \cap(Y \cap A=\varnothing\}, \\
\mathbf{P}\{X \cap A \neq \varnothing\} \mathbf{P}\{Y \cap A \neq \varnothing\}= & (1-\mathbf{P}\{X \cap A=\varnothing\})(1-\mathbf{P}\{Y \cap A=\varnothing\}) .
\end{aligned}
$$


Следовательно, необходимо доказать, что

$$
\mathbf{P}\{(X \cap A=\varnothing) \cap(Y \cap A=\varnothing)\}<\mathbf{P}\{X \cap A=\varnothing\} \mathbf{P}\{Y \cap A=\varnothing\} .
$$

Значения вероятностей равны

$$
\begin{array}{r}
\mathbf{P}\{X \cap A=\varnothing\}=\left(\begin{array}{c}
s-x \\
a
\end{array}\right)\left(\begin{array}{l}
s \\
a
\end{array}\right)^{-1}, \\
\mathbf{P}\{Y \cap A=\varnothing\}=\left(\begin{array}{c}
s-y \\
a
\end{array}\right)\left(\begin{array}{l}
s \\
a
\end{array}\right)^{-1}, \\
\mathbf{P}\{(X \cap A=\varnothing) \cap(Y \cap A=\varnothing)\}=\left(\begin{array}{c}
s-x-y \\
a
\end{array}\right)\left(\begin{array}{l}
s \\
a
\end{array}\right)^{-1}
\end{array}
$$

при $X \cap Y=\varnothing$, где

$$
s=|S|, \quad x=|X|, \quad y=|Y|, \quad a=|A| .
$$

Поэтому достаточно доказать справедливость неравенства

$$
\left(\begin{array}{c}
s-x-y \\
a
\end{array}\right)\left(\begin{array}{l}
s \\
a
\end{array}\right)^{-1}<\left(\begin{array}{c}
s-x \\
a
\end{array}\right)\left(\begin{array}{l}
s \\
a
\end{array}\right)^{-1}\left(\begin{array}{c}
s-y \\
a
\end{array}\right)\left(\begin{array}{l}
s \\
a
\end{array}\right)^{-1}
$$

или

$$
D_{y}=\left(\begin{array}{c}
s-x-y \\
a
\end{array}\right)\left(\begin{array}{c}
s-y \\
a
\end{array}\right)^{-1}<\left(\begin{array}{c}
s-x \\
a
\end{array}\right)\left(\begin{array}{l}
s \\
a
\end{array}\right)^{-1}=D_{0},
$$

где

$$
D_{i}=\left(\begin{array}{c}
s-x-i \\
a
\end{array}\right)\left(\begin{array}{c}
s-i \\
a
\end{array}\right)^{-1}, \quad i=0,1, \ldots, y .
$$

Но последовательность $D_{i}$ убывает при $i=0,1, \ldots, y$, так как

$$
\frac{D_{i}}{D_{i+1}}=\frac{s-x-i}{s-x-i-a} \frac{s-i-a}{s-i}=\frac{1-a /(s-i)}{1-a /(s-x-i)}>1 .
$$

Лемма доказана. вида:

Определим класс опорных множеств в поясе $S_{m-k, m-k+r}^{n}$ для $1 \leqslant r \leqslant k$ следующего

$$
A_{m-k, m-k+r}^{n}(\lambda)=\left\{A=\bigcup_{j=k-r}^{k} A_{m-j}: A_{m-j} \subset B_{m-j}^{n},\left|A_{m-j}\right|=a_{m-j}\right\}
$$

и

$$
a_{m-j}=\left\lceil\frac{\lambda}{g_{m-j}}\left(\begin{array}{c}
n \\
m-k+j
\end{array}\right)\right\rceil=\left\lceil\frac{\lambda}{g_{m-k+j}}\left(\begin{array}{c}
n \\
m-j
\end{array}\right)\right\rceil,
$$


где $\lambda-$ положительный параметр. Обозначим

$$
\begin{aligned}
\bar{G} & =\bar{G}\left(A_{m-k, m-k+r}^{n}(\lambda), S_{m-k, m}^{n}\right) \\
& =\frac{1}{\left|A_{m-k, m-k+r}^{n}(\lambda)\right|} \sum_{A \in A_{m-k, m-k+r}^{n}(\lambda)} G\left(A, S_{m-k, m}^{n}\right)
\end{aligned}
$$

среднее значение числа $\left(A, S_{m-k, m}^{n}\right)$-граничных вершин при случайном выборе опорного подмножества $A \in A_{m-k, m-k+r}^{n}(\lambda)$.

Лемма 5. Для $c_{\varepsilon}^{\prime} n \leqslant m \leqslant n / 2, k \leqslant m\left(1-c_{\varepsilon}^{\prime \prime}\right) / 2 u c_{\varepsilon}<\lambda<m / k-2-c_{\varepsilon} u \lambda=o(n)$ при $n \rightarrow \infty$ существует опорное подмножество $A_{\lambda} \in A_{m-k, m-k+r}^{n}(\lambda)$, для которого справедлива оченка числа граничных вершин

$$
\left|S_{m-r, m}^{n}\right|\left(1-e^{-\lambda}\right)\left(1-\frac{\lambda k}{m-2 k}\right) \lesssim \bar{G} \leqslant\left|G\left(A_{\lambda}, S_{m-k, m}^{n}\right)\right| .
$$

Доказательство. Существование опорного подмножества $A_{\lambda}$, имеющего число граничных вершин не меньше, чем среднее значение числа граничных вершин по всем опорным подмножествам из $A_{m-k, m-k+r}^{n}(\lambda)$, является очевидным. Для вычисления и оценивания среднего значения $\bar{G}\left(A_{m-k, m-k+r}^{n}(\lambda), S_{m-k, m}^{n}\right)$ перейдем к вероятностной модели. Положим, что независимо для каждого слоя $B_{m-j}^{n}$ случайно выбирается подмножество $A_{m-j}=A \cap B_{m-j}^{n}$ вершин опорного множества $A$, содержащее ровно $a_{m-j}$ вершин. Вероятность $\mathbf{P}\{A\}$ любого опорного множества $A \in A_{m-k, m-k+r}^{n}(\lambda)$ определяется следующим образом:

$$
\mathbf{P}\{A\}=\left|A_{m-k, m-k+r}^{n}(\lambda)\right|^{-1},
$$

где

$$
\left|A_{m-k, m-k+r}^{n}(\lambda)\right|=\prod_{j=k-r}^{k}\left(\begin{array}{c}
b_{m-j}^{n} \\
a
\end{array}\right)_{m-j}
$$

И

$$
b_{m-j}^{n}=\left|B_{m-j}^{n}\right|=\left(\begin{array}{c}
n \\
m-j
\end{array}\right) .
$$

Так как для любой вершины $\tilde{x} \in B_{m-j}^{n}$ по лемме $2 G\left(\tilde{x}, S_{m-k, m}^{n}\right) \in B_{m-k+j}^{n}$, то для любого $A \in A_{m-k, m-k+r}^{n}(\lambda)$ справедливо включение $G\left(A, S_{m-k, m}^{n}\right) \subset S_{m-r, m}^{n}$. Поэтому, вводя индикаторную функцию $\xi\left\{\tilde{x} \in G\left(A, S_{m-k, m}^{n}\right)\right\} \in\{0,1\}$ для события, состоящего в том, что вершина $\tilde{x}$ является $\left(A, S_{m-k, m}^{n}\right)$-граничной вершиной, получим представление

$$
\begin{aligned}
\bar{G} & =\sum_{A \in A_{m-k, m-k+r}^{n}(\lambda)} G\left(A, S_{m-k, m}^{n}\right) \mathbf{P}\{A\} \\
& =\sum_{A \in A_{m-k, m-k+r}^{n}(\lambda)} \sum_{\tilde{x} \in S_{m-r, m}^{n}} \xi\left\{\tilde{x} \in G\left(A, S_{m-k, m}^{n}\right)\right\} \mathbf{P}\{A\} \\
& =\sum_{\tilde{x} \in S_{m-r, m}^{n}} A \in A_{m-k, m-k+r}^{n}(\lambda) \\
& =\sum_{\tilde{x} \in S_{m-r, m}^{n}} \mathbf{P}\left\{\tilde{x} \in G\left(A, S_{m-k, m}^{n}\right)\right\} .
\end{aligned}
$$




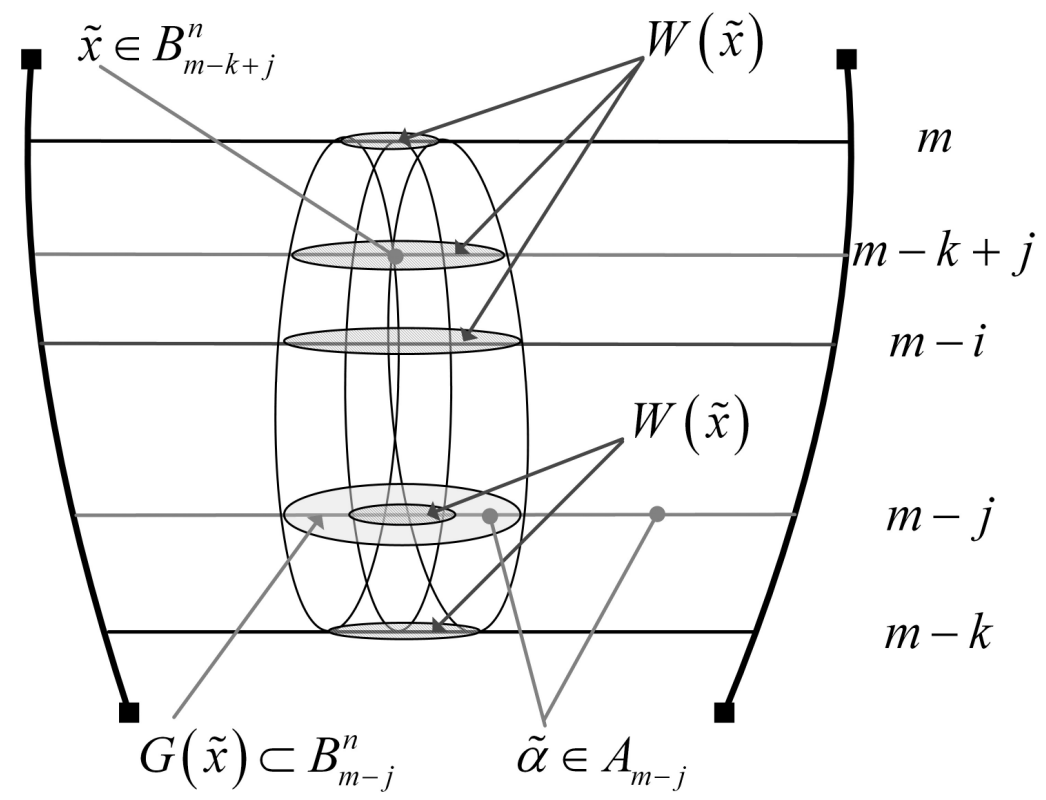

Рис. 2. $G\left(\tilde{x}, S_{m-k, m}^{n}\right)$-граничные вершины

Для любой вершины $\tilde{x} \in B_{m-k+j}^{n} \subset S_{m-r, m}^{n}$, где $k-r \leqslant j \leqslant k, G\left(\tilde{x}, S_{m-k, m}^{n}\right)$-граничные вершины принадлежат слою $B_{m-j}^{n}$ (см. рис. 2), поэтому

$$
\begin{aligned}
\mathbf{P}\left\{\tilde{x} \in G\left(A, S_{m-k, m}^{n}\right)\right\} & =\mathbf{P}\left\{\left(G\left(\tilde{x}, S_{m-k, m}^{n}\right) \cap A \neq \varnothing\right) \cap\left(W\left(\tilde{x}, S_{m-k, m}^{n}\right) \cap A=\varnothing\right)\right\} \\
& =\mathbf{P}\left\{\left(G\left(\tilde{x}, S_{m-k, m}^{n}\right) \cap A_{m-j} \neq \varnothing\right) \cap\left(W\left(\tilde{x}, S_{m-k, m}^{n}\right) \cap A=\varnothing\right)\right\} \\
& =\mathbf{P}\left\{\left(G\left(\tilde{x}, S_{m-k, m}^{n}\right) \cap A_{m-j} \neq \varnothing\right)\right\} \\
& -\mathbf{P}\left\{\left(G\left(\tilde{x}, S_{m-k, m}^{n}\right) \cap A_{m-j} \neq \varnothing\right) \cap\left(W\left(\tilde{x}, S_{m-k, m}^{n}\right) \cap A \neq \varnothing\right)\right\} .
\end{aligned}
$$

Так как

$$
A=\bigcup_{i=k-r}^{k} A_{m-i}
$$

и подмножества $A_{m-i} \subset B_{m-i}^{n}$ выбираются независимо, то для второго выражения справедлива оценка

$$
\begin{aligned}
\mathbf{P}_{2}= & \mathbf{P}\left\{\left(G\left(\tilde{x}, S_{m-k, m}^{n}\right) \cap A_{m-j} \neq \varnothing\right) \cap\left(W\left(\tilde{x}, S_{m-k, m}^{n}\right) \cap A \neq \varnothing\right)\right\} \\
\leqslant & \sum_{i=k-r}^{k} \mathbf{P}\left\{\left(G\left(\tilde{x}, S_{m-k, m}^{n}\right) \cap A_{m-j} \neq \varnothing\right) \cap\left(W\left(\tilde{x}, S_{m-k, m}^{n}\right) \cap A_{m-i} \neq \varnothing\right)\right\} \\
= & \mathbf{P}\left\{\left(G\left(\tilde{x}, S_{m-k, m}^{n}\right) \cap A_{m-j} \neq \varnothing\right) \cap\left(W\left(\tilde{x}, S_{m-k, m}^{n}\right) \cap A_{m-j} \neq \varnothing\right)\right\} \\
& \quad+\sum_{\substack{i=k-r \\
i \neq j}}^{k} \mathbf{P}\left\{G\left(\tilde{x}, S_{m-k, m}^{n}\right) \cap A_{m-j} \neq \varnothing\right\} \mathbf{P}\left\{W\left(\tilde{x}, S_{m-k, m}^{n}\right) \cap A_{m-i} \neq \varnothing\right\} .
\end{aligned}
$$


Из леммы 4, применяемой в слое $B_{m-j}^{n}$, то есть для множеств $S=B_{m-j}^{n}$, $X=G\left(\tilde{x}, S_{m-k, m}^{n}\right) \subset B_{m-j}, Y=W\left(\tilde{x}, S_{m-k, m}^{n}\right) \cap B_{m-j}, A=A_{m-j}$ получаем,что

$$
\begin{aligned}
\mathbf{P}\left\{\left(G\left(\tilde{x}, S_{m-k, m}^{n}\right) \cap A_{m-j} \neq \varnothing\right) \cap\left(W\left(\tilde{x}, S_{m-k, m}^{n}\right) \cap A_{m-j} \neq \varnothing\right)\right\} \\
\quad<\mathbf{P}\left\{G\left(\tilde{x}, S_{m-k, m}^{n}\right) \cap A_{m-j} \neq \varnothing\right\} \mathbf{P}\left\{W\left(\tilde{x}, S_{m-k, m}^{n}\right) \cap A_{m-j} \neq \varnothing\right\}
\end{aligned}
$$

при условии, что

$$
\| G\left(\tilde{x}, S_{m-k, m}^{n}\right)|+| W\left(\tilde{x}, S_{m-k, m}^{n}\right) \cap B_{m-j}|+| A_{m-j}|<| B_{m-j}^{n} \mid .
$$

Для выполнения этого условия достаточно, чтобы $\left|A_{m-j}\right|=o\left(\left|B_{m-j}^{n}\right|\right)$, то есть $\lambda=o(n)$ при $n \rightarrow \infty$. Поэтому

$$
\mathbf{P}_{2}<\mathbf{P}\left\{G\left(\tilde{x}, S_{m-k, m}^{n}\right) \cap A_{m-j} \neq \varnothing\right\} \sum_{i=k-r}^{k} \mathbf{P}\left\{W\left(\tilde{x}, S_{m-k, m}^{n}\right) \cap A_{m-i} \neq \varnothing\right\} .
$$

В силу выбора значения $a_{m-j}=\left|A_{m-j}\right|$ и п.2 леммы 3

$$
\begin{aligned}
\mathbf{P}\left\{G\left(\tilde{x}, S_{m-k, m}^{n}\right) \cap A_{m-j} \neq \varnothing\right\} & =1-\mathbf{P}\left\{G\left(\tilde{x}, S_{m-k, m}^{n}\right) \cap A_{m-j}=\varnothing\right\} \\
& =1-\left(\begin{array}{c}
b_{m-j}^{n}-g_{m-k+j} \\
a_{m-j}
\end{array}\right) /\left(\begin{array}{c}
b_{m-j}^{n} \\
a_{m-j}
\end{array}\right) \\
& \geqslant 1-\exp \left\{-\frac{a_{m-j}}{g_{m-k+j}} b_{m-j}^{n}\right\} \\
& \geqslant 1-\exp \left\{-\lambda \frac{b_{m-k+j}^{n} g_{m-k+j}}{b_{m-j}^{n} g_{m-j}}\right\}=1-e^{-\lambda} .
\end{aligned}
$$

Поэтому для $k-r \leqslant j \leqslant k$ и $\tilde{x} \in S_{m-r, m}^{n}$ получаем, что

$$
\begin{aligned}
\mathbf{P}\left\{\tilde{x} \in G\left(A, S_{m-k, m}^{n}\right)\right\} \geqslant & \mathbf{P}\left\{G\left(\tilde{x}, S_{m-k, m}^{n}\right) \cap A_{m-j} \neq \varnothing\right\} \\
& \times\left(1-\sum_{i=k-r}^{k} \mathbf{P}\left\{W\left(\tilde{x}, S_{m-k, m}^{n}\right) \cap A_{m-i} \neq \varnothing\right\}\right) \\
\geqslant & \left(1-e^{-\lambda}\right)\left(1-\sum_{i=k-r}^{k} \mathbf{P}\left\{W\left(\tilde{x}, S_{m-k, m}^{n}\right) \cap A_{m-i} \neq \varnothing\right\}\right) .
\end{aligned}
$$

Тогда для значения $\bar{G}$ справедлива следующая оценка снизу:

$$
\begin{aligned}
\bar{G} & =\sum_{\tilde{x} \in S_{m-r, m}^{n}} \mathbf{P}\left\{\tilde{x} \in G\left(A, S_{m-k, m}^{n}\right)\right\} \\
& \geqslant\left(1-e^{-\lambda}\right) \sum_{\tilde{x} \in S_{m-r, m}^{n}}\left(1-\sum_{i=k-r}^{k} \mathbf{P}\left\{W\left(\tilde{x}, S_{m-k, m}^{n}\right) \cap A_{m-i} \neq \varnothing\right\}\right) \\
& =\left(1-e^{-\lambda}\right)\left(\left|S_{m-r, m}^{n}\right|-\sum_{i=k-r}^{k} \sum_{\tilde{x} \in S_{m-r, m}^{n}} \mathbf{P}\left\{W\left(\tilde{x}, S_{m-k, m}^{n}\right) \cap A_{m-i} \neq \varnothing\right\}\right) .
\end{aligned}
$$


Для оценки сверху выражения $\sum_{\tilde{x} \in S_{m-r, m}^{n}} \mathbf{P}\left\{W\left(\tilde{x}, S_{m-k, m}^{n}\right) \cap A_{m-i} \neq \varnothing\right\}$ используем индикаторную функцию $\xi\{C\} \in\{0,1\}$ истинности события $C$

$$
\begin{aligned}
& \sum_{\tilde{x} \in S_{m-r, m}^{n}} \mathbf{P}\left\{W\left(\tilde{x}, S_{m-k, m}^{n}\right) \cap A_{m-i} \neq \varnothing\right\} \\
& =\sum_{\tilde{x} \in S_{m-r, m}^{n}} \sum_{A \in A_{m-k, m-k+r}^{n}(\lambda)} \mathbf{P}\{A\} \xi\left\{W\left(\tilde{x}, S_{m-k, m}^{n}\right) \cap A_{m-i} \neq \varnothing\right\} \\
& =\sum_{A \in A_{m-k, m-k+r}^{n}(\lambda)} \mathbf{P}\{A\} \sum_{\tilde{x} \in S_{m-r, m}^{n}} \xi\left\{\tilde{x} \in W\left(A_{m-i}, S_{m-k, m}^{n}\right)\right\} \\
& =\sum_{A \in A_{m-k, m-k+r}^{n}(\lambda)} \mathbf{P}\{A\}\left|W\left(A_{m-i}, S_{m-k, m}^{n}\right) \cap S_{m-r, m}^{n}\right| \leqslant\left|A_{m-i}\right| w_{m-i},
\end{aligned}
$$

так как

$$
\begin{gathered}
\xi\left\{W\left(\tilde{x}, S_{m-k, m}^{n}\right) \cap A_{m-i} \neq \varnothing\right\}=\xi\left\{\tilde{x} \in W\left(A_{m-i}, S_{m-k, m}^{n}\right)\right\}, \\
\left|W\left(A_{m-i}, S_{m-k, m}^{n}\right)\right| \leqslant\left|A_{m-i}\right| w_{m-i},
\end{gathered}
$$

где $w_{m-i}-$ число внутренних вершин в поясе $S_{m-k, m}^{n}$ для одной вершины из слоя $B_{m-i}^{n}$. Подставляя значение для $\left|A_{m-i}\right|$ и используя оценки леммы 3 , получаем, что

$$
\begin{aligned}
& \sum_{i=k-r}^{k}\left|A_{m-i}\right| w_{m-i} \leqslant \sum_{i=k-r}^{k}\left(\frac{\lambda}{g_{m-i}}\left(\begin{array}{c}
n \\
m-k+i
\end{array}\right)+1\right) \frac{k g_{m-i}}{m-2 k} \\
& =\frac{k}{m-2 k}\left(\lambda \sum_{i=k-r}^{k}\left(\begin{array}{c}
n \\
m-k+i
\end{array}\right)+\sum_{i=k-r}^{k} g_{m-i}\right) \\
& \leqslant \frac{k}{m-2 k}\left(\lambda\left|S_{m-r, m}^{n}\right|+\left(\begin{array}{c}
n \\
k+1
\end{array}\right)\right) \text {. }
\end{aligned}
$$

Для $c_{\varepsilon}^{\prime} n \leqslant m \leqslant n / 2$ и $k \leqslant m\left(1-c_{\varepsilon}^{\prime \prime} / 2\right)$ выполняется равенство $\left(\begin{array}{c}n \\ k+1\end{array}\right)=o\left(b_{m}^{n}\right)$, то есть

$$
\left(\begin{array}{c}
n \\
k+1
\end{array}\right)=o\left(\left|S_{m-r, m}^{n}\right|\right)
$$

при $n \rightarrow \infty$. Следовательно,

$$
\begin{aligned}
\bar{G} & \geqslant\left(1-e^{-\lambda}\right)\left(\left|S_{m-r, m}^{n}\right|-\frac{k}{m-2 k}\left(\lambda\left|S_{m-r, m}^{n}\right|+\left(\begin{array}{c}
n \\
k+1
\end{array}\right)\right)\right) \\
& =\left|S_{m-r, m}^{n}\right|\left(1-e^{-\lambda}\right)\left(1-\frac{\lambda k}{m-2 k}-\frac{k}{m-2 k}(1+o(1))\right) \\
& \gtrsim\left|S_{m-r, m}^{n}\right|\left(1-e^{-\lambda}\right)\left(1-\frac{\lambda k}{m-2 k}\right)
\end{aligned}
$$

в силу ограничений $c_{\varepsilon}<\lambda<m / k-2-c_{\varepsilon}$ и $\lambda=o(n)$ при $n \rightarrow \infty$. Лемма доказана.

Условие $k \leqslant m\left(1-c_{\varepsilon}^{\prime \prime}\right) / 2$ при $m \leqslant n / 2$ обеспечивает сравнимость числа граничных вершин с числом внутренних вершин для отдельной вершины опорного множества. Соответственно, при случайном выборе опорного множества $A \in A_{m-k, m-k+r}^{n}(\lambda)$, за счет 
уменьшения мощности опорного множества, регулируемой параметром $\lambda$, такое соотношение позволяет получить оценку среднего числа граничных вершин опорного множества, сравнимую с мощностью пояса.

Очевидно, что для параметра $r=r(n)$ достаточно выбирать такое значение, при котором выполняется соотношение

$$
\left|S_{m-k, m}^{n}\right| \sim\left|S_{m-r, m}^{n}\right|
$$

при $n \rightarrow \infty$ :

(a) $r(n)=k$, если $k=o(n)$,

(б) $r(n)=o(n)$ и $r(n) / \sqrt{n} \rightarrow \infty$, если $m \leqslant n / 2$,

(в) $r(n) \rightarrow \infty$, если $c_{\varepsilon}^{\prime} n \leqslant m \leqslant n / 2\left(1-c_{\varepsilon}\right)$.

Отметим, что если $m=o(n)$ при $n \rightarrow \infty$, то $\left|S_{m-k, m}^{n}\right| \sim\left|B_{m}^{n}\right|$, поэтому число граней в тупиковых комплексах для таких поясов куба $B^{n}$ определяется одним слоем $B_{m}^{n}$ и задача построения тупиковых комплексов с экстремальными характеристиками вырождается.

Отображение $\bar{\Psi}:\left(x_{1}, \ldots, x_{n}\right) \rightarrow\left(\bar{x}_{1}, \ldots, \bar{x}_{n}\right)$ для вершин единичного куба $B^{n}$ определяет автоморфизм, который сохраняет бинарное отношение смежности вершин в единичном кубе. При автоморфизме сохраняются структурные и метрические свойства для вершин и подмножеств единичного куба: слоев, поясов, интервалов. При этом для слоя $B_{m}^{n}$ справедливо равенство $\bar{\Psi}\left(B_{m}^{n}\right)=B_{n-m}^{n}$, где $0 \leqslant m \leqslant n$, и для пояса $S_{m-k, m}^{n}$ справедливо равенство $\bar{\Psi}\left(S_{m-k, m}^{n}\right)=S_{n-m, n-m+k}^{n}$, где $0 \leqslant k \leqslant m \leqslant n$. Следовательно, справедливы аналогичные утверждения и оценки для числа граничных вершин в поясе $S_{n-m, n-m+k}^{n}$ и из доказанных лемм 1-5 вытекает приведенная выше теорема 1.

Приведем следствия этой теоремы.

Следствие 1. Пусть $S=S_{m-k, m}^{n}$ или $S=S_{n-m, n-m+k}^{n}$, где $c_{\varepsilon} n \leqslant m \leqslant n / 2$ u $k=o(n)$, $k \rightarrow \infty$ при $n \rightarrow \infty$. Тогда в $S$ существует тупиковый комплекс $k$-мерных интервалов $T$ такой, что $l(T) \sim|S|$ при $n \rightarrow \infty$.

Доказательство. Положим $\lambda=\ln \left(m_{S} / k\right)$. Тогда выполняется условие $\lambda=o(n)$ и при этом $\lambda \rightarrow \infty$, то есть $1-e^{-\lambda} \sim 1$. В то же время

$$
\frac{\lambda k}{m_{S}-2 k}=\frac{m_{S}}{m_{S}-2 k} \frac{k}{m_{S}} \ln \frac{m_{S}}{k}=o(1)<1,
$$

так как $m_{S} /\left(m_{S}-2 k\right)=O(1)$ и $\left(k / m_{S}\right) \ln \left(m_{S} / k\right)=o(1)$. Следовательно,

$$
\left(1-e^{-\lambda}\right)\left(1-\lambda k /\left(m_{S}-2 k\right) \sim 1,\right.
$$

и, согласно теореме 1 , получаем $T$-тупиковый комплекс $k$-мерных интервалов, для которого $l(T) \sim|S|$ при $n \rightarrow \infty$.

Следствие 2. Если $k=o(n)$ u $k \rightarrow \infty$ при $n \rightarrow \infty$, то

$$
l_{T}(n, k) \sim 2^{n}
$$

то есть существует тупиковый комплекс $k$-мерных интервалов, в котором число интервалов асимптотически равно $2^{n}$. 
Доказательство. Положим $S_{i}=S_{m_{i}, m_{i}+k}^{n}$, где $m_{i}=\lfloor n / 2\rfloor+1+i(k+2)$ для $i$, $-m \leqslant i \leqslant m$ и $m=\lfloor n /(4 k)\rfloor$. Так как $m_{i}+k \leqslant\lfloor n / 2\rfloor-1$, если $i<0$ и $m_{i} \geqslant\lfloor n / 2\rfloor+1$, если $i \geqslant 0$, то при любом $i$ выполняются условия теоремы 1 и следствия 1 . Тогда в поясе $S_{i}$ существует тупиковый комплекс $k$-мерных интервалов $T_{i}$, в котором число интервалов асимптотически равно $\left|S_{i}\right|$. При объединении поясов $S=\bigcup_{-m \leqslant i \leqslant m} S_{i}$ каждое подмножество $S_{i}$ является компонентой связности, следовательно, объединение тупиковых комплексов $T=\bigcup_{-m \leqslant i \leqslant m} T_{i}$ будет тупиковым комплексом $k$-мерных интервалов. Число интервалов в комплексе $T$

$$
\begin{aligned}
l(T) & =\sum_{-m \leqslant i \leqslant m} l\left(T_{i}\right) \gtrsim \sum_{-m \leqslant i \leqslant m}\left|S_{i}\right| \sim 2^{n}-\sum_{-m \leqslant i \leqslant m}\left(\begin{array}{c}
n \\
\lfloor n / 2\rfloor+(k+2) i
\end{array}\right) \\
& \sim 2^{n}-\frac{1}{k+2} 2^{n} \sim 2^{n},
\end{aligned}
$$

если $k=o(n)$ и $k \rightarrow \infty$ при $n \rightarrow \infty$ (см. с. 279 в [13]). Заметим, что в случае, когда $k / \sqrt{n} \rightarrow \infty$, достаточно двух компонент $S_{-1}$ и $S_{0}$, так как $\left|S_{-1}\right|+\left|S_{0}\right| \sim 2^{n}$ при $n \rightarrow \infty$.

Следствие 3. Пусть $S=S_{m-k, m}^{n}$ или $S=S_{n-m, n-m+k}^{n}$, где

$$
c_{\varepsilon} n \leqslant m_{S} \leqslant n / 2, \quad c_{\varepsilon}^{\prime} n \leqslant k \leqslant m_{S} / 2\left(1-c_{\varepsilon}^{\prime \prime}\right)
$$

u $k / m_{S} \sim x$ при $n \rightarrow \infty$. Тогда в поясе $S$ существует тупиковый комплекс $k$-мерных интервалов $T$ такой, что

$$
l(T) \gtrsim|S| \varphi\left(\lambda_{0}, x\right)
$$

при $n \rightarrow \infty$, где

$$
\varphi(\lambda, x)=\left(1-e^{-\lambda}\right)\left(1-\frac{\lambda x}{1-2 x}\right),
$$

$u$ значение $\lambda_{0}$ выбирается из условия максимизации по $\lambda$ функиии $\varphi(\lambda, x)$ при $0<\lambda<1 / x-2$.

При наложенных условиях на $m$ и $k$ утверждение следствия 3 вытекает непосредственно из теоремы 1 , так как выполняется соотношение $0<\varphi(\lambda, x)<1$ при условии, что $0<x<1 / 2$ и $0<\lambda<1 / x-2$.

В табл. 1 представлены расчетные значения $\lambda_{0}$ и $\varphi_{0}=\varphi\left(\lambda_{0}, x\right)$ в зависимости от значения $x=k / m_{S}$.

Следствие 4. Если $c_{\varepsilon}^{\prime} n \leqslant k \leqslant n\left(1-c_{\varepsilon}^{\prime \prime}\right) / 4$ при $n \rightarrow \infty$, то

$$
l_{T}(n, k) \geqslant 2^{n},
$$

то есть существует тупиковый комплекс $k$-мерных интервалов, в котором число интервалов по порядку равно $2^{n}$.

Доказательство. Пусть $S=S_{m-k, m}^{n}$ или $S=S_{n-m, n-m+k}^{n}$, где $m=[n / 2-(t / 2) \sqrt{n}]$ и $0 \leqslant t \leqslant C$, где $C-$ некоторая постоянная. Тогда (см. с. 215 в [4])

$$
\begin{aligned}
|S| & \sim 2^{n-1}-\left|S_{\lfloor n / 2\rfloor-m,\lfloor n / 2\rfloor}^{n}\right| \\
& \sim 2^{n-1}-2^{n}(\Phi(0)-\Phi(-t))=2^{n} \Phi(-t) \geqslant 2^{n} .
\end{aligned}
$$

Если $t=o(1)$ или $m=\lfloor n / 2+o(n)\rfloor$, то $\Phi(-t) \sim \Phi(0)=1 / 2$ и $|S| \sim 2^{n-1}$. Поэтому при $k \leqslant(m / 2)\left(1-c_{\varepsilon}^{\prime \prime} \leqslant n\left(1-c_{\varepsilon}^{\prime \prime}\right) / 4\right.$ требуемое утверждение вытекает из следствия 3. 
Таблица 1.

\begin{tabular}{ccc|ccc|ccc}
$x$ & $\lambda_{0}$ & $\varphi_{0}$ & $x$ & $\lambda_{0}$ & $\varphi_{0}$ & $x$ & $\lambda_{0}$ & $\varphi_{0}$ \\
\hline & & & 0,17 & 1,2800 & 0,4839 & 0,34 & 0,4200 & 0,1899 \\
0,01 & 4,5475 & 0,9434 & 0,18 & 1,2081 & 0,4629 & 0,35 & 0,3861 & 0,1760 \\
0,02 & 3,8112 & 0,9002 & 0,19 & 1,1391 & 0,4425 & 0,36 & 0,3537 & 0,1624 \\
0,03 & 3,3650 & 0,8617 & 0,20 & 1,0737 & 0,4226 & 0,37 & 0,3222 & 0,1491 \\
0,04 & 3,0400 & 0,8263 & 0,21 & 1,0115 & 0,4032 & 0,38 & 0,2922 & 0,1361 \\
0,05 & 2,7856 & 0,7931 & 0,22 & 0,9528 & 0,3843 & 0,39 & 0,2631 & 0,1234 \\
0,06 & 2,5725 & 0,7616 & 0,23 & 0,8969 & 0,3659 & 0,40 & 0,2351 & 0,1110 \\
0,07 & 2,3887 & 0,7316 & 0,24 & 0,8431 & 0,3479 & 0,41 & 0,2079 & 0,0988 \\
0,08 & 2,2269 & 0,7029 & 0,25 & 0,7922 & 0,3304 & 0,42 & 0,1817 & 0,0869 \\
0,09 & 2,0831 & 0,6753 & 0,26 & 0,7434 & 0,3133 & 0,43 & 0,1563 & 0,0752 \\
0,10 & 1,9525 & 0,6486 & 0,27 & 0,6967 & 0,2965 & 0,44 & 0,1318 & 0,0638 \\
0,11 & 1,8325 & 0,6229 & 0,28 & 0,6519 & 0,2802 & 0,45 & 0,1080 & 0,0526 \\
0,12 & 1,7237 & 0,5979 & 0,29 & 0,6094 & 0,2643 & 0,46 & 0,0851 & 0,0416 \\
0,13 & 1,6237 & 0,5738 & 0,30 & 0,5683 & 0,2487 & 0,47 & 0,0628 & 0,0309 \\
0,14 & 1,5287 & 0,5503 & 0,31 & 0,5289 & 0,2335 & 0,48 & 0,0412 & 0,0204 \\
0,15 & 1,4412 & 0,5276 & 0,32 & 0,4911 & 0,2186 & 0,49 & 0,0203 & 0,0101 \\
0,16 & 1,3575 & 0,5054 & 0,33 & 0,4547 & 0,2041 & & &
\end{tabular}

Теорема 2. Для любого тупикового комплекса интервалов в единичном кубе $B^{n}$ число интервалов размерности не менее $k$ не превосходит $2^{n}(1-k /(2(n+1))$.

Доказательство. Пусть для тупикового комплекса интервалов $T=\left\{I_{j}, j=1, \ldots, l\right\}$ в единичном кубе $B^{n}$

$N_{T, k}^{+}=\bigcup_{I \in T: \operatorname{dim}(I) \geqslant k} N_{I}-$ подмножество вершин $N_{T}$, которые покрываются интервалами размерности не менее $k$;

$C_{T, k}^{+}=C_{T} \cap N_{T, k}^{+}-$подмножество собственных вершин, в которое входит по одной собственной вершине для каждого интервала размерности не менее $k$;

$\left|C_{T, k}^{+}\right|-$число интервалов размерности не менее $k$ в тупиковом комплексе $T$.

Если $\tilde{\alpha} \in N_{T, k}^{+}$, то

$$
\left|C_{T} \cap S_{1}(\tilde{\alpha})\right| \leqslant n-k+1,
$$

где $S_{1}(\tilde{\alpha})$ - шар Хэмминга радиуса 1 с центром в вершине $\tilde{\alpha}$. В этом случае интервал размерности не менее $k$, который покрывает вершину $\tilde{\alpha}$, покрывает еще не менее $k$ вершин в $S_{1}(\tilde{\alpha})$, которые не могут быть собственными. Если $\tilde{\alpha} \in B^{n} \backslash N_{T, k}^{+}$, то

$$
\left|C_{T} \cap S_{1}(\tilde{\alpha})\right| \leqslant\left|S_{1}(\tilde{\alpha})\right|=n+1 .
$$

Поэтому

$$
\begin{aligned}
(n+1)\left|C_{T}\right| & =\sum_{\tilde{\alpha} \in B^{n}}\left|C_{T} \cap S_{1}(\tilde{\alpha})\right|=\sum_{\tilde{\alpha} \in N_{T, k}^{+}}\left|C_{T} \cap S_{1}(\tilde{\alpha})\right|+\sum_{\tilde{\alpha} \in B^{n} \backslash N_{T, k}^{+}}\left|C_{T} \cap S_{1}(\tilde{\alpha})\right| \\
& \leqslant(n-k+1)\left|N_{T, k}^{+}\right|+(n+1)\left|B^{n} \backslash N_{T, k}^{+}\right|, \\
\left|C_{T, k}^{+}\right| & \leqslant\left|C_{T}\right| \leqslant\left(1-\frac{k}{n+1}\right)\left|N_{T, k}^{+}\right|+\left|B^{n} \backslash N_{T, k}^{+}\right|=2^{n}-\frac{k}{n+1}\left|N_{T, k}^{+}\right| .
\end{aligned}
$$


Так как $C_{T, k}^{+} \subseteq N_{T, k}^{+} \subseteq N_{T} \subseteq B^{n}$, то

$$
\left|C_{T, k}^{+}\right| \leqslant\left|N_{T, k}^{+}\right| \leqslant 2^{n}
$$

и, используя неравенство $\min (x, y) \leqslant(x+y) / 2$, получаем, что

$$
\begin{aligned}
\left|C_{T, k}^{+}\right| & \leqslant \min \left\{\left|N_{T, k}^{+}\right|, 2^{n}-\frac{k}{n+1}\left|N_{T, k}^{+}\right|\right\} \leqslant \frac{1}{2}\left\{2^{n}+\left(1-\frac{k}{n+1}\right)\left|N_{T, k}^{+}\right|\right\} \\
& \leqslant \frac{1}{2}\left\{2^{n}+2^{n}\left(1-\frac{k}{n+1}\right)\right\}=2^{n}\left(1-\frac{k}{2(n+1)}\right) .
\end{aligned}
$$

Теорема доказана.

Следствие 5. При $k \geqslant c(n+1)$ максимальное число интервалов размерности не менее $k$ в тупиковом комплексе единичного куба $B^{n}$ не превосходит $(1-c / 2) 2^{n}$.

\section{5. Оценки числа тупиковых комплексов}

Для собственной вершины $\tilde{x} \in C_{T}$ тупикового комплекса интервалов $T$ будем обозначать через $I_{T, \tilde{x}}$ интервал, покрывающий эту собственную вершину.

Пусть $T \in T_{m-k_{0}, m}^{n}-$ произвольный тупиковый комплекс $k_{0}$-мерных интервалов в поясе $S_{m-k_{0}, m}^{n}$ и параметры $p, k$ удовлетворяют неравенству $0<p<k<k_{0}$. Для каждой собственной вершины $\tilde{x} \in C_{T} \cap S_{m-p, m}^{n}$ определим пучок интервалов $P_{\tau}(\tilde{x})$ (см. рис. 3 )

$$
P_{\tau}(\tilde{x})=\left\{I: \tilde{\delta}_{\min }(I) \in B_{m-k}^{n} \cap I\left(\tilde{\delta}_{\min }\left(I_{T, \tilde{x}}\right), \tilde{x}\right), \tilde{\delta}_{\max }(I)=\tilde{\delta}_{\max }\left(I_{T, \tilde{x}}\right)\right\},
$$

где $\tilde{\delta}_{\min }(I)$ и $\tilde{\delta}_{\max }(I)$ обозначают соответственно минимальную и максимальную вершины произвольного интервала $I$ в $B^{n}$.

Будем рассматривать комплексы интервалов вида $\left\{I_{j}: I_{j} \in P_{\tau}\left(\tilde{x}_{j}\right), \tilde{x}_{j} \in C_{T} \cap S_{m-p, m}^{n}\right\}$, то есть для вершин $\tilde{x} \in C_{T} \cap S_{m-p, m}^{n}$ в комплекс входит по одному интервалу из пучка интервалов $P_{\tau}(\tilde{x})$.

Множество различных комплексов интервалов, построенных по пучкам интервалов $P_{\tau}(\tilde{x})$ для собственных вершин из $C_{T} \cap S_{m-p, m}^{n}$ тупикового комплекса $T \in T_{m-k_{0}, m}^{n}$, будем обозначать $\Omega_{\tau}^{n}\left(T, C_{T}, S_{m-k_{0}, m}^{n}, p, k\right)$.

Лемма 6. Множество $\Omega_{\tau}^{n}\left(T, C_{T}, S_{m-k_{0}, m}^{n}, p, k\right) \subseteq T_{m-k, m}^{n}$, то есть представляет собой подмножество тупиковых комплексов $k$-мерных интервалов в поясе $S_{m-k, m}^{n}$. Неравенство

$$
\left|P_{\tau}(\tilde{x})\right| \geqslant\left(\begin{array}{c}
k_{0}-p \\
k-p
\end{array}\right)
$$

справедливо для любой вершины $\tilde{x} \in C_{T} \cap S_{m-p, m}^{n}, u$

$$
\left|\Omega_{\tau}^{n}\left(T, C_{T}, S_{m-k_{0}, m}^{n}, p, k\right)\right| \geqslant\left(\begin{array}{c}
k_{0}-p \\
k-p
\end{array}\right)^{\left|C_{T} \cap S_{m-p, m}^{n}\right|} .
$$

Доказательство. Для любой вершины $\tilde{x} \in C_{T} \cap S_{m-p, m}^{n}$ любой интервал $I \in P_{\tau}(\tilde{x})$ является максимальным интервалом, так как $\tilde{\delta}_{\min }(I) \in B_{m-k}^{n}, \tilde{\delta}_{\max }(I) \in B_{m}^{n}$ и вне $S_{m-k, m}^{n}$ 


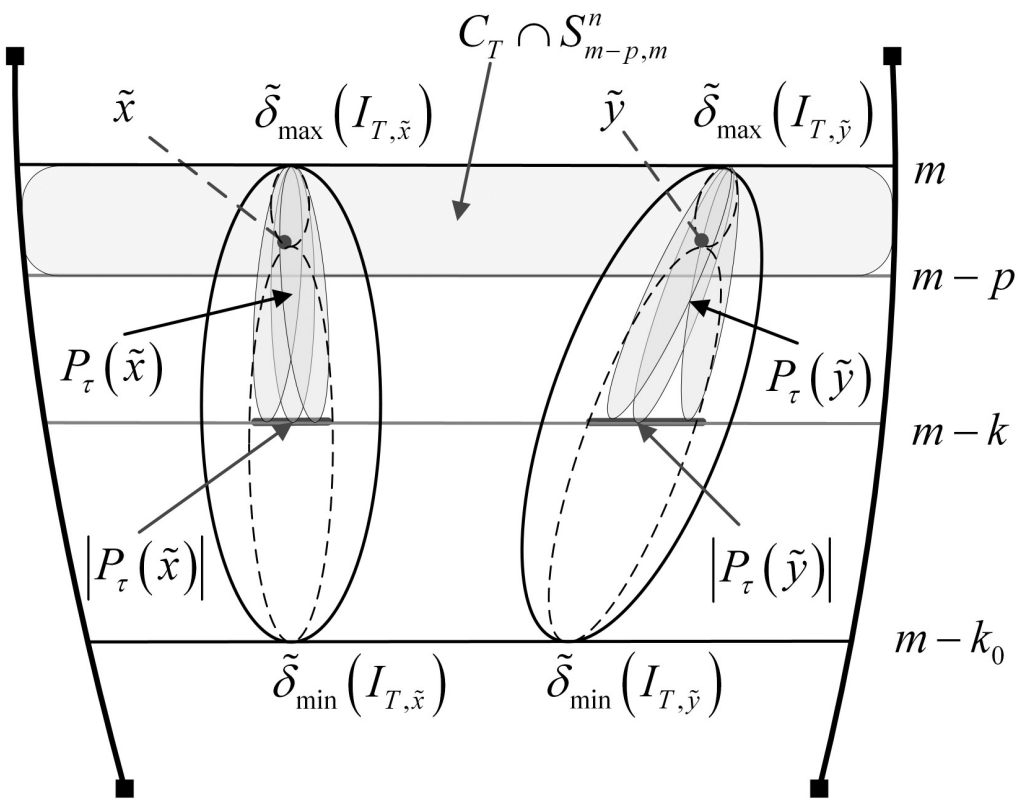

Рис. 3. Пучок интервалов $P_{\tau}(\tilde{x})$

нет ни одной вершины, покрываемой интервалами комплекса. При этом любой интервал $I \in P_{\tau}(\tilde{x})$ содержит вершину $\tilde{x}$ и $N_{\tau}(\tilde{x})=\bigcup_{I \in P_{\tau}}(\tilde{x}) I \subset I_{T, \tilde{x}}$, то есть множество вершин, покрываемых интервалами из пучка $P_{\tau}(\tilde{x})$, содержится в интервале $I_{T, \tilde{x}}$. Поэтому $\tilde{x} \notin \bigcup_{\tilde{y} \in C_{T}} \backslash\{\tilde{x}\} N_{\tau}(\tilde{y})$, так как $\tilde{x} \notin \bigcup_{\tilde{y} \in C_{T}} \backslash\{\tilde{x}\} I_{T, \tilde{y}}$, и для любого комплекса интервалов вершина $\tilde{x} \in C_{T} \cap S_{m-p, m}^{n}$ является собственной, так как покрывается одним интервалом из пучка $P_{\tau}(\tilde{x})$, который включается в комплекса. Это означает, что любой комплекс интервалов из $\Omega_{\tau}^{n}\left(T, C_{T}, S_{m-k_{0}, m}^{n}, p, k\right)$ является тупиковым комплексом интервалов из $T_{m-k, m}^{n}$.

Докажем второе неравенство. Заметим, что $\left|P_{\tau}(\tilde{x})\right|=\left|B_{m-k}^{n} \cap I\left(\tilde{\delta}_{\min }\left(I_{T, \tilde{x}}\right), \tilde{x}\right)\right|$ для любой вершины $\tilde{x} \in C_{T} \cap S_{m-p, m}^{n}$. Если $\tilde{x} \in B_{m-j}^{n} \subset S_{m-p, m}^{n}$, где $0 \leqslant j \leqslant p$, то интервал $I\left(\tilde{\delta}_{\min }\left(I_{T, \tilde{x}}\right), \tilde{x}\right)$ имеет размерность $(m-j)-\left(m-k_{0}\right)=\left(k_{0}-j\right)$, а пересечение этого интервала со слоем $B_{m-k}^{n}$ является $\left(k_{0}-k\right)$ слоем этого интервала. Поэтому для вершины $\tilde{x} \in B_{m-j}^{n}$, где $0 \leqslant j \leqslant p$, справедливы соотношения

$$
\left|P_{\tau}(\tilde{x})\right|=\left|B_{m-k}^{n} \cap I\left(\tilde{\delta}_{\min }\left(I_{T, \tilde{x}}\right), \tilde{x}\right)\right|=\left(\begin{array}{c}
k_{0}-j \\
k_{0}-k
\end{array}\right) \geqslant\left(\begin{array}{c}
k_{0}-p \\
k_{0}-k
\end{array}\right)=\left(\begin{array}{c}
k_{0}-p \\
k-p
\end{array}\right) .
$$

Тогда из построения множества комплексов следует, что

$$
\left|\Omega_{\tau}^{n}\left(T, C_{T}, S_{m-k_{0}, m}^{n}, p, k\right)\right|=\prod_{\tilde{x} \in C_{T} \cap S_{m-p, m}^{n}}\left|P_{\tau}(\tilde{x})\right| \geqslant\left(\begin{array}{c}
k_{0}-p \\
k-p
\end{array}\right)^{\left|C_{T} \cap S_{m-p, m}^{n}\right|} .
$$

Лемма доказана. 
Теорема 3. Пусть $c_{\varepsilon} n \leqslant m \leqslant n / 2, c_{\varepsilon}^{\prime} n \leqslant k_{0} \leqslant m\left(1-c_{\varepsilon}^{\prime \prime}\right) / 2 u k_{0} / m=x$. Если $n \rightarrow \infty$, $k<k_{0} u k / \sqrt{n} \rightarrow \infty$, mo

$$
\log \left|T_{m-k, m}^{n}\right|=\log \left|T_{n-m, n-m+k}^{n}\right| \gtrsim\left|S_{m-k, m}^{n}\right| k_{0} H\left(k / k_{0}\right) \varphi(\lambda, x),
$$

где $\varphi(\lambda, x)=\left(1-e^{-\lambda}\right)(1-\lambda x /(1-2 x))$.

Доказательство. Нижняя оценка для числа тупиковых комплексов получается при построении множества тупиковых комплексов $\Omega_{\tau}^{n}\left(T, C_{T}, S_{m-k_{0}, m}^{n}, p, k\right) \subset T_{m-k, m}^{n}$ по тупиковому комплексу $T \in T_{m-k_{0}, m}^{n}$, имеющему максимальное число интервалов. В силу следствия 3 при указанных значениях параметров $m$ и $k_{0}$ в поясе $S_{m-k_{0}, m}^{n}$ существует тупиковый комплекс $k_{0}$-мерных интервалов $T$ такой, что

$$
\left|C_{T}\right|=l(T) \gtrsim\left|S_{m-k_{0}, m}^{n}\right| \varphi(\lambda, x)
$$

при $n \rightarrow \infty$. А из леммы 6 следует, что для параметров $p$ и $k$, удовлетворяющих неравенству $0<p<k<k_{0}$, справедливо неравенство

$$
\log \left|T_{m-k, m}^{n}\right| \geqslant\left|C_{T} \cap S_{m-p, m}^{n}\right| \log \left(\begin{array}{c}
k_{0}-p \\
k-p
\end{array}\right) .
$$

Положим $p=k / \ln (k / \sqrt{n})$. Тогда $p=o\left(k_{0}\right), p=o(k)$ и $p / \sqrt{n} \rightarrow \infty$ при $n \rightarrow \infty$. Поэтому, во-первых, выполняется соотношение $\left|S_{m-p, m}^{n}\right| \sim\left|S_{m-k_{0}, m}^{n}\right|$ и, следовательно,

$$
\left|C_{T} \cap S_{m-p, m}^{n}\right| \sim\left|C_{T} \cap S_{m-k_{0}, m}^{n}\right|=\left|C_{T}\right| .
$$

Во-вторых, выполняется соотношение

$$
\log \left(\begin{array}{c}
k_{0}-p \\
k-p
\end{array}\right) \sim \log \left(\begin{array}{c}
k_{0} \\
k
\end{array}\right) \sim k_{0} H\left(k / k_{0}\right)
$$

при $n \rightarrow \infty$ (см. с. 280 в [13]). Теорема доказана.

Следствие 6. Если $n \rightarrow \infty,\lfloor n / 2\rfloor \leqslant m \leqslant\lfloor n / 2\rfloor+O(\sqrt{n})$ u $k \sim x m$, где $0<x<1 / 2$, то

$$
\log \left|T_{m-k, m}^{n}\right|=\log \left|T_{n-m, n-m+k}^{n}\right| \asymp n 2^{n} .
$$

Доказательство. Положим $k_{0}=\lfloor y m\rfloor$, где $0<x<y<1 / 2$. Из теоремы 3, при подстановке значений параметров $k$ и $k_{0}$, следует оценка

$$
\log \left|T_{m-k, m}^{n}\right| \gtrsim m\left|S_{m-k, m}^{n}\right| y \varphi(\lambda, y) t H(x / y)
$$

при $n \rightarrow \infty$, где $0<\lambda<1 / y-2$. Учитывая, что $\left|S_{m-k, m}^{n}\right| \asymp 2^{n}$ и $m \sim n / 2$, получаем, что

$$
\log \left|T_{m-k, m}^{n}\right| \gtrsim \frac{n}{2}\left|S_{m-k, m}^{n}\right| C(x, y, \lambda) \gtrsim n 2^{n},
$$

где

$$
C(x, y, \lambda)=y \varphi(\lambda, y) H(x / y), \quad 0<x<y<1 / 2, \quad 0<\lambda<1 / y-2 .
$$

Следствие 7. Пусть $T(n, k)$ - число тупиковых комплексов $k$-мерных граней в кубе $B^{n}$. Тогда $\log T(n, k) \asymp n 2^{n}$ при $n \rightarrow \infty$, если $k=[c n]$, где $0<c<1 / 4$. 
Таблица 2.

\begin{tabular}{cccc|cccc}
$x$ & $y_{0}$ & $\lambda_{0}$ & $c(x)$ & $x$ & $y_{0}$ & $\lambda_{0}$ & $c(x)$ \\
\hline 0,01 & 0,08584 & 2,14085 & 0,48966 & 0,26 & 0,34275 & 0,41031 & 0,81364 \\
0,02 & 0,10634 & 1,87624 & 0,75023 & 0,27 & 0,35027 & 0,38510 & 0,76443 \\
0,03 & 0,12261 & 1,69732 & 0,93165 & 0,28 & 0,35768 & 0,36102 & 0,71533 \\
0,04 & 0,13687 & 1,55774 & 1,06441 & 0,29 & 0,36504 & 0,33772 & 0,66652 \\
0,05 & 0,14985 & 1,44244 & 1,16281 & 0,30 & 0,37229 & 0,31534 & 0,61821 \\
0,06 & 0,16200 & 1,34277 & 1,23519 & 0,31 & 0,37946 & 0,29379 & 0,57055 \\
0,07 & 0,17347 & 1,25537 & 1,28706 & 0,32 & 0,38652 & 0,27313 & 0,52373 \\
0,08 & 0,18445 & 1,17698 & 1,32229 & 0,33 & 0,39352 & 0,25313 & 0,47791 \\
0,09 & 0,19503 & 1,10570 & 1,34378 & 0,34 & 0,40042 & 0,23389 & 0,43323 \\
0,10 & 0,20524 & 1,04086 & 1,35378 & 0,35 & 0,40727 & 0,21524 & 0,38985 \\
0,11 & 0,21518 & 0,98071 & 1,35406 & 0,36 & 0,41401 & 0,19731 & 0,34792 \\
0,12 & 0,22483 & 0,92531 & 1,34607 & 0,37 & 0,42068 & 0,17993 & 0,30757 \\
0,13 & 0,23428 & 0,87335 & 1,33102 & 0,38 & 0,42727 & 0,16320 & 0,26894 \\
0,14 & 0,24352 & 0,82486 & 1,30992 & 0,39 & 0,43379 & 0,14694 & 0,23218 \\
0,15 & 0,25255 & 0,77934 & 1,28363 & 0,40 & 0,44022 & 0,13131 & 0,19743 \\
0,16 & 0,26144 & 0,73644 & 1,25291 & 0,41 & 0,44659 & 0,11610 & 0,16483 \\
0,17 & 0,27014 & 0,69604 & 1,21840 & 0,42 & 0,45287 & 0,10139 & 0,13454 \\
0,18 & 0,27870 & 0,65778 & 1,18067 & 0,43 & 0,45907 & 0,08721 & 0,10672 \\
0,19 & 0,28710 & 0,62142 & 1,14022 & 0,44 & 0,46519 & 0,07346 & 0,08153 \\
0,20 & 0,29540 & 0,58688 & 1,09750 & 0,45 & 0,47124 & 0,06009 & 0,05918 \\
0,21 & 0,30359 & 0,55388 & 1,05292 & 0,46 & 0,47719 & 0,04723 & 0,03988 \\
0,22 & 0,31162 & 0,52257 & 1,00684 & 0,47 & 0,48307 & 0,03473 & 0,02388 \\
0,23 & 0,31956 & 0,49263 & 0,95959 & 0,48 & 0,48884 & 0,02268 & 0,01152 \\
0,24 & 0,32740 & 0,46400 & 0,91145 & 0,49 & 0,49450 & 0,01109 & 0,00327 \\
0,25 & 0,33512 & 0,43657 & 0,86272 & & & &
\end{tabular}

Теорема 4. При достаточно больших $n$ справедлива оценка $T(n) \geqslant\left(2^{2^{n}}\right)^{c n}$, где $c>1,355 \cdot 2^{-5}$.

Доказательство. Для получения нижней оценки числа $T(n)$ рассмотрим два пояса $S_{m-1-k, m-1}^{n}$ и $S_{n-m+1, n-m+1+k}^{n}$ в кубе $B^{n}$ для параметров $m=\lfloor n / 2\rfloor$ и $k / m \sim x$, где $0<x<1 / 2$. Тогда

$$
\left|S_{m-1-k, m-1}^{n}\right|=\left|S_{n-m+1, n-m+1+k}^{n}\right| \sim 2^{n-1},
$$

и справедлива оценка (см. следствие 6)

$$
\log \left|T_{m-1-k, m-1}^{n}\right|=\log \left|T_{n-m+1, n-m+1+k}^{n}\right| \gtrsim n 2^{n-2} C(x, y, \lambda),
$$

где

$$
C(x, y, \lambda)=y \varphi(\lambda, y) H(x / y), \quad 0<x<y<1 / 2, \quad 0<\lambda<1 / y-2 .
$$

Значения параметров $x, y$ и $\lambda$ выбираются из условия максимизации функции $C(x, y, \lambda)$ в области $\{(x, y, \lambda): 0<x<y<1 / 2,0<\lambda<1 / y-2\}$. Численная оптимизация позволяет получить оценку $C(0,1052,0,2104,1,0087)>1,3550 \cdot 2^{-4}$.

Так как множества вершин $S_{m-1-k, m-1}^{n}$ и $S_{n-m+1, n-m+1+k}^{n}$ являются компонентами связности в кубе $B^{n}$ (см. с. 117 в [2]), то

$$
T(n) \geqslant\left|T_{m-1-k, m-1}^{n}\right| \times\left|T_{n-m+1, n-m+1+k}^{n}\right| .
$$


Поэтому для $k \sim x m \approx 0,0526 n$ получаем, что

$$
\log T(n) \geqslant \log \left|T_{m-1-k, m-1}^{n}\right|+\log \left|T_{n-m+1, n-m+1+k}^{n}\right|>1,355 n 2^{n-5} .
$$

Теорема доказана.

Оценка числа тупиковых комплексов $k$-мерных граней в кубе $B^{n}$ для $k \sim x n / 2$ и $0<x<1 / 2$ имеет вид $\log T(n, k)>c(x) n 2^{n-5}$, где

$$
c(x)=\max _{\substack{0<x<y<1 / 2 \\ 0<\lambda<1 / y-2}} 2^{4} C(x, y, \lambda) .
$$

В табл. 2 представлены расчетные значения для $c(x)$.

\section{Список литературы}

1. Журавлев Ю. И., Алгоритмы построения минимальных д.н.ф. В сб.: Дискретная математика и математические вопросы кибернетики, 1. Наука, Москва, 1974, с. 67-98.

2. Васильев Ю. Л., Глаголев В. В., Метрические свойства дизъюнктивных нормальных форм. В сб.: Дискретная математика и математические вопросы кибернетики, 1. Наука, Москва, 1974, c. 99-148.

3. Сапоженко А. А., Чухров И. П., Минимизация булевых функций в классе дизъюнктивных нормальных форм. В сб.: Итоги науки и техники. Серия Теория вероятностей, Математическая статистика, Теоретическая кибернетика. ВИНИТИ АН СССР, Москва, 1987, с. 68-116.

4. Яблонский С. В., Введение в дискретную математику. Высшая школа, Москва, 2003.

5. Журавлев Ю. И., Оценка для числа тупиковых д.н.ф. функций алгебры логики. Сибирский матем. журнал (1962) 3, №5, 802-804.

6. Васильев Ю. Л., К вопросу о числе минимальных и тупиковых дизъюнктивных нормальных форм. Дискретный анализ (1964), № 2, 3-9.

7. Сапоженко А. А., О наибольшей длине тупиковой дизьюнктивной нормальной формы у почти всех булевых функций. Математические заметки (1968) 4, №6, 649-658.

8. Сапоженко А. А., Дизъюнктивные нормальные формыл. МГУ, Москва, 1975.

9. Чухров И. П., Оценка числовых характеристик поясковых функций. В сб.: Тезисы докл. V Всесоюзной конф. по проблемам теоретической кибернетики, Новосибирск, 1980, с. 179-180.

10. Чухров И. П., О числе тупиковых дизъюнктивных нормальных форм. Доклады АН СССР (1982) 262, №6, 1329-1332.

11. Яблонский С. В., К вопросу об оценке длины тупиковых дизъюнктивных нормальных форм. Проблемы кибернетики (1962) 7, 229-230.

12. Глаголев В. В., О длине тупиковой дизъюнктивной нормальной формы. Математические заметки (1967) 2, №6, 665-672.

13. Гаврилов Г. П., Сапоженко А. А., Задачи и упражнения по дискретной математике. Физматлит, Москва, 2005.

Статья поступила 8.09.2010. 